\title{
Combination of in-situ diffraction experiments and acoustic emission testing to understand the compression behaviour of Mg-Y-Zn alloys containing LPSO phase under different loading conditions.
}

G. Garcés ${ }^{\mathrm{a}, *}$, K. Máthis ${ }^{\mathrm{b}}$, J. Medina $^{\mathrm{a}}$, K. Horváth ${ }^{\mathrm{b}}$, D. Drozdenko ${ }^{\mathrm{b}}$, E. Oñorbe ${ }^{\mathrm{c}}$, P. Dobroñ ${ }^{\mathrm{b}}, \mathrm{P}$. Pérez $^{\text {a }}$, M. Klaus ${ }^{\text {d }}$ P. Adeva ${ }^{\mathrm{a}}$

aDepartment of Physical Metallurgy, CENIM-CSIC, Avenida Gregorio del Amo 8, E-28040 Madrid, Spain

${ }^{b}$ Department of Physics of Materials, Faculty of Mathematics and Physics, Charles University, KeKarlovu 5, 12116 Praha 2, Czech Republic

${ }^{c}$ Department of technology. Division of materials of energy interest, CIEMAT. Avenida Complutense, 40, 28040 Madrid, Spain

${ }^{\mathrm{d} H e l m h o l t z-Z e n t r u m ~ B e r l i n, ~ A l b e r t-E i n s t e i n-S t r a ß e ~ 15, ~} 12489$ Berlin, Germany.

*corresponding author. Tel +34-91-553-8900; fax: +34-91-534-7425;

E-mail address: ggarces@,cenim.csic.es

\section{Abstract}

The effect of the orientation of the non-recrystallized grains (non-DRX) and the LPSO phase on plasticity in extruded Mg97Y2Zn1 alloy with a bimodal grain structure have been studied in-situ using the combination of synchrotron diffraction and acoustic emission techniques during compression tests. The adaptive sequential k-means (ASK) procedure was applied to analyze the acoustic emission signal. This method can successful separate the signal for each possible deformation systems. Combining both techniques the deformation mechanisms that take place during the compression tests under different loading directions have been distinguish. Independently of the loading direction, the beginning of the macroscopic plasticity is always controlled by the activation of basal slip system in the DRX grains. However, highly oriented non-DRX grains and LPSO phase have a strong influence over the compressive deformation of the MgY2Zn1 alloy. The basal planes in the non-DRX grains were oriented parallel to the extrusion direction (ED). Thus, the activation of the $\{10 \overline{1} 2\}\langle 10 \overline{11}\rangle$ extension twinning was found to be significant only in the ED mode. In the other two loading cases, TD and 45, the activation of non-basal slip systems has been detected. On the other hand, the reinforcing effect of the 
elongated LPSO phase is the most effective, if the loading axis is aligned with the fiber direction (extrusion direction) since the load transfer mechanism is more effective. In this loading case, the LPSO can be plastically deformed due to kinking. This deformation mode has been isolated by the ASK procedure and characterized by in-situ scanning electron microscopy.

\section{Keywords:}

A. Strengthening mechanisms; Twinning; B. Anisotropic material; C. Mechanical testing; InSitu testing 


\section{Introduction}

Extruded magnesium alloys containing Long-Period Stacking Ordered (LPSO) phase are in the forefront of the current magnesium research, since they provide high mechanical strength and good creep resistance at intermediate temperatures (Inoue et al., 2001; Itoi et al., 2008; Garces et al., 2007; Yamasaki et al., 2005; Kawamura et al., 2006; Hagihara et al., 2010; Kawamura et al., 2007; Yamasaki et al., 2011; Garces et al., 2012a; Oñorbe et al., 2013). The LPSO phase is a long range stacking of basal planes with periodic enrichment of transition metals and yttrium or rare earth with a chemical order in periodic basal plane. LPSO-phases reinforce the magnesium matrix due to their higher Young's modulus (Tane et al., 2013) and enhance grain refinement during thermo-mechanical processing (Hagihara et al., 2010; Oñorbe et al., 2012; Oñorbe et al., 2011).

The as-extruded microstructure of $\mathrm{Mg}-\mathrm{Y}-\mathrm{Zn}$ alloy is characterized by a mixture of (i) fine, equiaxed and randomly oriented dynamically recrystallized $\alpha-\mathrm{Mg}$ grains (DRX grains); (ii) coarse elongated hot-worked $\alpha-\mathrm{Mg}$ grains having their basal planes oriented parallel to the extrusion direction (ED) - non-DRX grains, and (iii) fibre-shaped LPSO-phase elongated along ED (Yamasaki et al., 2011; Hirano et al., 2010; Garces et al. 2015a). Yamasaki et al. (Yamasaki et al., 2011) studied the tensile behavior of these multi-phase $\mathrm{Mg}-\mathrm{Zn}-\mathrm{Y}$ alloys concluding that the fine magnesium grains contribute to enhancement of the ductility of the alloy, while the coarse textured $\alpha-\mathrm{Mg}$ grains as well as the LPSO phase cause an increment in the mechanical strength. However, during compression along ED, the coarse non-DRX grains are softer in comparison to the DRX grains. The reason is given by the orientation of the non-DRX grains, which favor the activation of the $\{10 \overline{1} 2\}\langle 10 \overline{11}\rangle$ extension twinning system (Christian and Mahajan, 1995; Yoo, 1981; Kleiner and Uggowitzer, 2004). Garces et al. (Garces et al., 2015a) have studied the elasto-plastic behavior during compression along ED using synchrotron diffraction experiment in the $\mathrm{MgY}_{2} \mathrm{Zn}_{1}$ alloy. The beginning of plasticity in this alloy is controlled by the activation of the basal slip system in the DRX grains because their volume 
fraction is higher than that of the worked coarse grains. Moreover, the $\{10 \overline{1} 2\}\langle 10 \overline{1}\rangle$ extension twinning also takes place and controls plasticity above the yield point changing the macroscopic texture of the alloy. However, they suggested that extension twinning could occur in non-DRX grains before the macroscopic yield strength. In order to clarify the exact role of the non-DRX grains in the plasticity of $\mathrm{Mg}_{97} \mathrm{Y}_{2} \mathrm{Zn}_{1}$ alloy under compression, in this work, we applied acoustic emission (AE) technique in addition to the synchrotron radiation diffraction (SRD) experiments. The main advantage of this experimental method is its sensitivity to twin nucleation and high time resolution in order of microseconds. Moreover, the adaptive sequential k-means (ASK) method proposed by Pomponi and Vinogradov (Pomponi and Vinogradov, 2013) will be use to separate the individual contribution of each deformation system to the global AE signal. Thus, the combination of diffraction experiments, sensitive on the twin growth, and the AE technique can lead to a throughout characterization of the twinning process. Furthermore, the specimens were compressed along various directions (extrusion (ED), transversal (TD) and direction tilted by $45^{\circ}$ from ED to TD) in order to reveal how the specimens' orientations influences both the extension twinning and the reinforcing effect of the LPSO phase.

\section{Experimental procedure}

\subsection{Materials}

The $\mathrm{Mg}_{97} \mathrm{Y}_{2} \mathrm{Zn}_{1}\left(\right.$ at.\%) and $\mathrm{Mg}_{88} \mathrm{Y}_{7} \mathrm{Zn}_{5}($ at.\%) alloys were fabricated by melting in an electric resistance furnace using high purity $\mathrm{Mg}, \mathrm{Y}$ and $\mathrm{Zn}$ elements and a $\mathrm{Mg}-\mathrm{Y}$ master alloy. The molten alloy was cast in a cylindrical steel mold with a diameter of $42 \mathrm{~mm}$. The round billets of the $\mathrm{Mg}_{97} \mathrm{Y}_{2} \mathrm{Zn}_{1}$ alloy were extruded through a rectangular die of a section $4 \mathrm{~mm}$ by $20 \mathrm{~mm}$ at $350{ }^{\circ} \mathrm{C}$ with a reduction ratio of $18: 1$. On the other hand, the $\mathrm{MgY}_{7} \mathrm{Zn}_{5}$ alloy were extruded through a circular die with a diameter of $20 \mathrm{~mm}$ at $450{ }^{\circ} \mathrm{C}$ with a reduction ratio of $4: 1$. 


\subsection{Microstructural and mechanical characterization}

Scanning electron microscope (SEM) and Electron backscatter diffraction (EBSD) technique was used to perform detailed microstructure analyses before and after compression loading. Microstructure investigation was performed using JEOL JSM 6500F. Quantitative image analysis was carried out to quantify the volume fraction of the LPSO phase. 20 SEM images in the Back-scattered (BS) mode have been obtained. In the BS mode the magnesium matrix is completely black and the LPSO phase white. The LPSO phase is evaluated using the software Fiji ImageJ (Schindelin et al., 2012).

In addition, orientation patterns of the samples after loading were collected using SEM FEI Quanta 200 FEG equipped with EDAX/TSL EBSD systems. The mapping was carried out on longitudinal section of the samples with a step size of $0.4 \mu \mathrm{m}$ and an acceleration voltage of 15 $\mathrm{kV}$. The data acquisition software program TSL-OIM Analysis 7.0 was used to process the EBSD data. The sample surface for EBSD mapping was ground, polished by diamond pastes down to $0.25 \mu \mathrm{m}$ particle size and subsequently subjected for Ar ion milling in the Gatan Precision Ion Polishing System (PIPS). Ion bombarding was performed under the angle of $4^{\circ}$ using the voltage of $2.5 \mathrm{keV}$ for $3.5 \mathrm{~h}$.

Micro-computed tomography $(\mathrm{CT})$ measurements was performed to disclose the $3 \mathrm{D}$ shape, distribution and interconnectivity of the LPSO phase. $\mu$-CT measurements were performed by means of synchrotron radiation at BAM line (BESSY II). X-ray photons of $E=35 \mathrm{keV}$ were selected from the polychromatic spectrum using a double multilayer monochromator. The sample was positioned at $5 \mathrm{~mm}$ from the scintillator, taking 3000 radioscopic images within $180^{\circ}$ rotation and acquisition time of 3 seconds. Flat fields were took every 50 expositions for later corrections in the reconstruction. The pixel size of the CCD camera was $0.4361 \times 0.4361$ microns. The reconstruction was done slice by slice using filtered projection algorithm for parallel beam projections. Then, with ANKA phase program, phase contrast was obtained. 
Then, the 3D tomogram of the sample was formed by stacking the reconstructed 2D CTslices. The segmentation and analysis of this volume was done with Avizo 3D software.

Texture analysis was performed by the back-Schulz reflection method, using a SIEMENS TM Kristalloflex D5000 X-ray diffractometer equipped with an Eulerian cradle. $\beta$-filtered $\mathrm{Cu}-\mathrm{K}_{\alpha}$ radiation was used for this analysis. Experimental (0002), $\{10 \overline{1} 0\},\{10 \overline{1} 1\},\{10 \overline{1} 2\},\{10 \overline{1} 3\}$ and $\{11 \overline{2} 0\}$ pole figures were obtained by measuring over a polar angle range of $0^{\circ}$ to $75^{\circ}$ with $5^{\mathrm{o}}$ steps and a measuring time of $30 \mathrm{~s}$ per orientation. The beam optics include primary Soller slits of $2.3^{\circ}$ divergence, incident-beam crossed slits of $1^{\circ}$ divergence and receiving slits of $0.1^{\circ}$ divergence before the detector. Defocusing was corrected by using a standard random $\mathrm{Mg}$ powder sample. Orientation distribution functions (ODFs) describing texture were computed from these pole figures by the series expansion method, considering the Z-axis to be parallel to the extrusion direction. From the ODF and the symmetry of the system, pole figures can be recalculated for the whole polar angle range.

The mechanical properties of the alloy in all loading conditions were evaluated by compression testing. Samples with a square cross-section of $4 \mathrm{~mm}$ on edge and with a length of $8 \mathrm{~mm}$ were cut by electrical discharge machining for loading in three compression mode: along ED, TD and direction tilted by $45^{\circ}$ from ED to TD. Compression tests at room temperature were carried out using an universal testing machine at a strain rate of $10^{-3} \mathrm{~s}^{-1}$.

\subsection{In-situ X-ray synchrotron radiation diffraction in the $\mathrm{MgY}_{2} \mathrm{Zn}_{1}$ alloy}

In-situ synchrotron diffraction experiments were carried out on the beamline EDDI at BESSY, Berlin, Germany. The prismatic specimens were compressed uniaxially using a servo-hydraulic rig WALER \& BAI company capable of a $20 \mathrm{kN}$ maximum load at room temperature (RT). Compression tests were performed in a load control mode in the elastic regime and in a strain control mode in the plastic regime.

Samples with a square cross-section of $4 \mathrm{~mm}$ on edge and with a length of $8 \mathrm{~mm}$ were cut by electrical discharge machining for loading in three compression mode: along ED, TD and 
direction tilted by $45^{\circ}$ from ED to TD (hereafter, ED, TD and $45^{\circ}$ modes, respectively). Deformation modes were chosen to follow deformation mechanisms during compression as a function of both, orientation of the non-DRX grains and fibers of LPSO phases. Therefore, Fig.1(a-c) illustrates the orientation of the fibers of LPSO phase and non-DRX coarse magnesium grains (prolongation of grains and orientation of c-axis of hcp lattice) with respect to sample geometry for three compression modes. When the compression axis is parallel to ED, the non-DRX grains are elongated along the compression axis and their basal planes are parallel to compression axis. Under this condition, the activation of the $\{10 \overline{1} 2\}\langle 10 \overline{11}\rangle$ extension twinning system is expected, in agreement with a previous studies (Garces et al., 2015 a). When the compression axis is parallel to TD, significant fraction of non-DRX grains have their c-axis perpendicular to the compression direction (initial texture). However, the twinned volume is expected to be smaller in comparison to the ED mode. Finally, in $45^{\circ}$ mode, the majority of non-DRX grains cannot undergo twinning. In the leftover grains some twinning can take place (see further the EBSD maps), but the basal $\langle a>$-slip will be the dominant deformation mechanism.

The energy range of the synchrotron white beam was from 10 to $135 \mathrm{keV}$. The diffraction angle was around $2 \theta=9.74^{\circ}$. The resulting gauge was a rhomboid prism of $0.5 \times 0.1 \times 3.3 \mathrm{~mm}^{3}$. The gauge volume was always positioned in the center of the compression sample.

The sample was tilted within the scattering plane between $\psi=0^{\circ}$ (axial direction) and $\psi=90^{\circ}$ (radial direction), where $\psi$ is defined as the angle between the scattering vector and the extrusion axis. A cylindrical co-ordinate system (axial and radial directions) was adopted due to the symmetry of the extrusion process. When the extrusion axis was parallel to the scattering vector q, the axial strain component was measured, and when the extrusion axis was perpendicular to q, the radial component was measured. 
The use of a white beam allowed the collection of the entire diffraction pattern. Individual diffraction peaks were fitted with a Gaussian function to determine the peak position and peak intensity. This analysis was carried out using the provided software package at the EDDI beamline (Genzel et al., 2007). The resulting information allowed lattice spacing values, $d$, to be obtained using Bragg's law:

$d^{h k l}=\frac{h c}{2 \sin \theta E^{h k l}}$

where $h$ is Planck's constant, $c$ is the speed of light, $\theta$ is the diffraction angle and $E$ is the peak energy. The elastic strain in the principal direction $i$ (axial or radial) is given by:

$\varepsilon_{i}^{h k l}=\frac{d_{i}^{h k l}}{d_{0}^{h k l}}-1=\frac{E_{0}^{h k l}}{E_{i}^{h k l}}-1$

where $d_{0}$ is the lattice spacing of the stress-free value and $d_{i}$ is the lattice spacing measured when the diffraction vector is parallel to the principal direction i. The peak value, when compression stress is zero, is selected as the stress-free value, $E_{0}$, since initial micro-residual stresses are not developed during extrusion process (Garces et al. 2015b).

The $\{10 \overline{1} 0\},(0002),\{10 \overline{1} 1\},\{10 \overline{1} 2\}$ and $\{11 \overline{2} 0\}$ diffraction peaks of the Mg phase and the $\{4 \overline{22} 8\}$ diffraction peak from the 18R LPSO were collected and fitted during compression.

\subsubsection{In-situ X-ray synchrotron diffraction in the $\mathrm{MgY}_{7} \mathrm{Zn}_{5}$ alloy}

Moreover, for comparison purpose, in-situ synchrotron diffraction experiments were also carried out in a $\mathrm{MgY}_{7} \mathrm{Zn}_{5}($ at.\%) alloy, with an almost fully LPSO microstructure (Garces at al., 2014), on the beamline P07 at DESY, Berlin, Germany. Since the high absorption of the LPSO phase for low energies, it was not possible to obtain good diffraction peaks in EDDI beamline below $40 \mathrm{KeV}$. The high energy of the P07 beamline of PETRA III, $100 \mathrm{KeV}$, overcomes this problem and allows to obtain diffraction patterns with reliable diffraction peaks.

The compression samples are cylinders with a diameter $5 \mathrm{~mm}$ and length of $10 \mathrm{~mm}$ machined in the along ED, TD and direction tilted by $45^{\circ}$ from ED to TD (hereafter, ED, TD and $45^{\circ}$ modes, respectively). They were tested using a Bähr $805 \mathrm{~A} / \mathrm{D}$ dilatometer at a strain rate of $10^{-3} \mathrm{~s}^{-1}$. The 
gauge volume is defined by $1 \times 1 \times 5 \mathrm{~mm}^{3}$ (beam section $\mathrm{x}$ cylinder diameter). The diffraction patterns were recorded using an exposure time of $0.5 \mathrm{~s}$ by a Perkin-Elmer XRD 1622 flatpanel detector with an array of $2048^{2}$ pixel, with an effective pixel size of $200 \times 200 \mu \mathrm{m}^{2}$. The wavelength was $0.0124 \mathrm{~nm}$. LaB6 was used as a reference to calibrate the acquired diffraction spectra. The detector-to-sample distance was $1789 \mathrm{~mm}$. Conventional $2 \theta$ diffraction profiles were obtained by azimuthal integration of the Debye-Scherrer rings (over a range $10^{\circ}\left( \pm 5^{\circ}\right)$ centered about $0^{\circ}$ and $180^{\circ}$ in the axial direction and about $90^{\circ}$ and $270^{\circ}$ in the radial direction) in the axial and radial direction. The fitting of the diffraction peaks was carried out with the FIT2D software (Hammersley., 1997) using a gaussian function. The elastic strain for each orientation can be calculated by the relative shift in the position of the diffraction peak. Using equation (1) the elastic strain is given by:

$\varepsilon_{i}^{h k l}=\frac{\sin \theta_{0}^{h k l}}{\sin \theta_{i}^{h k l}}-1$

where $\theta_{i}^{h k l}$ and $\theta_{0}^{h k l}$ are the diffraction angle of the hkl plane in the stressed and stress-free crystal.

The $\{4 \overline{22} 2\},\{4 \overline{22} 5\},(00018),\{4 \overline{22} 8\},\{30 \overline{3} 12\},\{4 \overline{22} 10\}$ and $\{60 \overline{6} 0\}$ diffraction peaks have been evaluated for the 18R LPSO structure.

\subsection{Acoustic Emission}

The AE measurements were performed during separate deformation tests, realized at the same condition as in the case of the in-situ X-ray diffraction, including the specimen size. PCI-2 acquisition board (Mistras Corp.) was used for recording of the AE response. The Micro30Stype sensor, having a flat response between 50 and $650 \mathrm{kHz}$ was connected to the specimens using a clamp. Vacuum grease was applied between the sensor and the specimen in order to ensure good acoustic contact. The AE signal was pre-amplified using a 2-4-6 type bandpass $(100-1200 \mathrm{kHz})$ preamplifier, giving a gain of $60 \mathrm{~dB}$. The measurements took place in the socalled data streaming mode, when the raw signal is recorded. The evaluation of the AE signal 
was done by adaptive sequential $k$-means (ASK) analysis (Pomponi and Vinogradov, 2013) described in detail in Section3.3.

\subsection{In-situ scanning electron microscopy (SEM)}

To confirm findings from SRD and AE measurements in-situ SEM observations (SE and BSD imaging) were provided by Zeiss Auriga SEM. The surface of the samples having dimension of $3.3 \times 2.6 \times 2.3 \mathrm{~mm}^{3}$ were ground on 4000 grit $\mathrm{SiC}$ paper and then polished using diamond paste of $1 / 4 \mu \mathrm{m}$ particle size. The compression tests along ED and TD were performed inside the SEM chamber by means of a MTESTQuattro (Materials Testing System, ADMET) deformation stage. Based on the $\mathrm{AE}$ and diffraction results, particular stress levels were chosen, at which the SEM images were acquired.

\section{Results}

\subsection{Initial microstructure and texture}

Figure $2 \mathrm{a}$ shows the 3D SEM image of the microstructure of the extruded $\mathrm{Mg}_{97} \mathrm{Y}_{2} \mathrm{Zn}_{1}(\mathrm{at} . \%)$ alloy in the three principal direction of the extruded plate: ED, TD and normal direction (ND). The LPSO phase is distributed as long fibers aligned with ED. Figure $2 b$ shows the segmented and colored $\mu \mathrm{CT}$-tomograms of the extruded alloy. The magnesium matrix has been made transparent and only the LPSO-phase is observed. 3D image presents elongated and independent LPSO fibers about 200-300 $\mu \mathrm{m}$ length. The volume fraction of the LPSO-phase calculated from BS-SEM images and tomography was 19 and 25\%, respectively. The magnesium matrix shows a bimodal grain structure with dynamically-recrystallized (DRX) grains having an average size of $0.9 \mu \mathrm{m}$ and non-recrystallized (non-DRX) grains elongated in ED with a grain length of $50 \mu \mathrm{m}$ and a width about $10 \mu \mathrm{m}$ (Figure $2 \mathrm{c}$ ). The volume fraction of each grain type, measured by image analysis of micrographs (Figure 2c), was determined as 59 $\%$ and $22 \%$ for the DRX and the non-DRX areas, respectively. 
Figure $2(\mathrm{~d}, \mathrm{e})$ shows the recalculated $\{10 \overline{1} 0\}$ and (0002) pole figures of the Mg phase measured perpendicularly to ED. The alloy shows a strong fibre texture with the basal plane parallel to ED. It has been reported that the majority of the intensity contributing to the fiber texture is given by the non-DRX grains, which have their basal planes oriented parallel to ED(Yamasaki et al., 2011; Garces et al., 2015a; Garces et al., 2015c). It should be noted, that among non-DRX grains, most of the grains have their basal planes oriented parallel to ED and TD (hcp lattice marked as "1" in Fig. 1) and leftover has their basal planes oriented parallel to ED and ND (hcp lattice marked as "2" and " 3 " in Fig. 1).

Figure 3(a-d) shows the axial and radial patterns obtained from samples before compression in ED and TD, respectively. Owing to the used diffraction geometry and the initial texture of the specimens, the intensities of the (0002) and $\{10 \overline{1} 0\}$ peaks are different for each compression case and they depend on the sample orientation. For example, the $\{10 \overline{1} 0\}$ peak has the highest intensity for ED sample in axial direction. In contrast, it is small for TD (cf. Figs. 2and 3). As it will be shown in the following subchapter, these two peaks can be used for characterization of extension twinning.

Figure 4(a-c) shows the Debye-Scherrer rings obtained in the $\mathrm{MgY}_{7} \mathrm{Zn}_{5}$ alloy before the compression tests in the ED, TD and $45^{\circ}$ modes. Arrows in Fig. 4 a indicate the axial and radial direction, respectively. The inner ring (indicated with white arrows) corresponds to the (0003) diffraction peak (Garces et al., 2015b). In the ED mode, the maximum is located along the radial direction. After the extrusion, the basal plane of the 18R LPSO structure is oriented parallel to the extrusion direction in agreement with Garces et al.(Garces et al., 2014). After integration, diffraction patterns along the axial direction as a function of $2 \theta$ are obtained (Figure $4 d$ ). In the ED mode, owing to the intense fiber texture of the alloy, the diffraction peak corresponding to the $\{4 \overline{22} 2\}$ plane ( $\{10 \overline{1} 0\}$ plane in the magnesium hexagonal structure) and the $\{60 \overline{6} 0\}$ plane exhibit the highest intensity. In contrast, the intensity of the diffraction peaks corresponding to 
(0003) and (00018) plane is very low. This behavior is inverted in the diffraction pattern obtained in the TD direction.

3.2. Deformation experiments with in-situ X-ray synchrotron diffraction in a Mg alloy containing LPSO fibers (MgY2Zn1 alloy).

Continuous deformation curves obtained in the universal testing machine and discontinuous deformation curves obtained in the beamline during in-situ experiments for all three compression modes are presented in Fig. 5a and b, respectively. In figure 5a, the elastic part has been removed since the small dimension of the sample, it was impossible the use of extensometer. The compression behaviour obtained in the beamline in consistent with those obtained in the continuous compression tests. The compression strain varies between 7 and $10 \%$. It is obvious that the mechanical properties are influenced by the sample orientation. The highest yield strength value was found for the sample compressed in the ED direction $(\sim 340$ $\mathrm{MPa}$ ), whereas the lowest value corresponded to the sample compressed in the TD direction $(\sim 250 \mathrm{MPa})$.

The evolution of internal elastic strains (calculated using Eq. (2)) for the magnesium diffraction peaks in the axial directions as a function of the applied stress is shown in Figure6(a-c). It has been demonstrated in previous study of the extruded $\mathrm{MgY}_{2} \mathrm{Zn}_{1}$ alloy (Garces et al., 2015a) that axial and radial elastic strains give analogous information. Therefore, for simplicity, only axial data are plotted. The compression stress-strain curves are also plotted in Fig.6(a-c), allowing the easy comparison of overall sample strains and specific-planar strains. Moreover, Fig. 6(ac) also shows the changes of the integrated intensities of the $\{10 \overline{1} 0\}$ and (0002) peaks as a function of the applied stress, in order to characterize the twin growth (Gharghouri et al., 1999). For comparison purpose, the integrated intensity as a function of the applied stress of the $\{10 \overline{1} 0\}$ and (0002) diffraction peaks in logarithmic scale for the three compression modes are also presented in Fig. 6d. 
Finally, Figure 6 e shows the evolution of elastic strain for the $\{4 \overline{22} 8\}$ diffraction peak of the LPSO phase in the axial directions as a function of the applied stress for sample compressed in the ED, TD and $45^{\circ}$ modes.

The evolution of the internal deformations of the magnesium and LPSO phases will be described in detail for each compression mode in the following sections.

\subsubsection{Compression along extrusion direction (ED)}

The evolution of elastic strains in the axial direction as a function of the applied stress for the ED sample (Fig. 6a) indicates that in the elastic regime, i.e. below the macroscopic yield stress ( $\sim 340 \mathrm{MPa})$, the evolution of internal strains in the $\mathrm{Mg}$ phase has a linear character with identical slopes for all diffracted peaks, which represent the Young's modulus. Mg alloys are known to be elastically isotropic and no differences of elastic strains between different orientations have been observed in the elastic regime (Agnew et al., 2006). The Young's modulus at room temperature (RT) is estimated as $\mathrm{E}_{\mathrm{Mg}}=46 \mathrm{GPa}$ which is in agreement with experimental values for magnesium alloys (44.7GPa) (Brandes and Brooks, 1997). The evolution of internal strain for the $\{4 \overline{22} 8\}$ diffraction peak of the LPSO phase (Fig. 6e) also exhibits linear behavior up to $350 \mathrm{MPa}$ and the Young's modulus at RT is estimated as ELPSO= $58 \mathrm{GPa}$. Thus, the Young's modulus is higher than that for the Mg matrix. It is important to note that it is not possible to fit the diffraction peak above this stress due to the high increase in the intensity of the (0002) diffraction peak of the magnesium phase which overlaps with the small peak of the LPSO phase. For this reason, in-situ diffraction experiments in an alloy with only the 18R LPSO phase were also carried out (section 3.4) to support these observations.

The loss of the linearity of the elastic internal strains is normally connected with plastic deformation. At $340 \mathrm{MPa}$, the grains with their $\{10 \overline{1} 1\}$ - and $\{10 \overline{1} 2\}$ - planes oriented parallel to the stress/extrusion direction lose their elastic linearity. These grains are well oriented for activation of the basal slip system(Agnew et al., 2001), and it is likely that macroscopic yielding occurs by the operation of this system. 
However, the (0002) diffraction peaks lose elastic linearity at $250 \mathrm{MPa}$, see Fig. 6a. The Schmid factor for the activation of basal slip is zero for these grains. Thus, the plastic deformation must be related to the activation of non-basal slip or extension twinning. The evolution of the integrated intensities of the $\{10 \overline{1} 0\}$ and (0002) peaks during the compression test is shown in Fig.6a. As it was shown first by Ghargouri et al.(Gharghouri et al., 1999), the reorientation of the lattice by $86^{\circ}$ during extension twinning leads to an increase in the intensity of (0002) diffraction peak in the axial direction and the concurrent decrease in the intensity of the $\{10 \overline{1} 0\}$ diffraction peak. As it is obvious from Fig. 6d, the intensity of the (0002) peak, in logarithmic scale, increases slightly in microyield region (250 MPa - $340 \mathrm{MPa})$ followed by a large increment above $365 \mathrm{MPa}$. This indicates a significant role of the extension twinning in strain accommodation above this stress level. Concurrently, the evolution of internal strains of the (0002) diffraction peak (Fig. 6a) change abruptly again. In contrast to the microyield region, where the internal strains increases (from -5252 to $-4062 \mu$ strains) here a linear decrease with a slope lower than that in the elastic regime can be observed.

The lattice strains on $\{10 \overline{1} 1\}$ - and $\{10 \overline{1} 2\}$-planes begin to deviate from the straight line of the elastic response in the vicinity of the macroscopic yield. Since both planes have a high Schmid factor for the basal slip ( 0.36 and 0.43 , respectively), the effect can be explained in terms of strain accommodation by slip of basal $<$ a $>$ dislocations (Agnew et al., 2006). Nevertheless, in the case of the plane $\{10 \overline{1} 1\}$ the contribution of the prismatic $<a>$ slip also cannot be excluded $\left(\mathrm{SF}_{\text {prism }}=0.34\right)$. After yielding, the elastic strains corresponding to $\{10 \overline{1} 1\}-$ and $\{10 \overline{1} 2\}-$ diffraction peaks are relaxed towards positive values from -6872 and $-6465 \mu$ strains at $340 \mathrm{MPa}$ to $-5313 \mu$ strains and $-4431 \mu$ strains at $363 \mathrm{MPa}$, respectively. Then, their values remain constant as a function of the applied stress.

On the other hand, the $\{10 \overline{1} 0\}$ oriented grains have to accumulate larger portion of load, since both the basal slip and twinning are difficult to be active for the given loading direction. 
Therefore, the lattice strain deviates to the left side above the yield point from the linear response (cf. Fig. 6a).

\subsubsection{Compression along transverse direction}

In the $\mathrm{TD}$, the evolution of internal strains of all the diffracted peaks is linear with applied stress until the macroscopic yield stress (260 MPa), without a trace of microyielding (cf. Fig. 6b). The Young's modulus at RT is estimated as $\mathrm{E}_{\mathrm{Mg}}=45 \mathrm{GPa}$. Linear internal strain-stress dependence for the $\{4 \overline{22} 8\}$ diffraction peak of the LPSO phase (Fig. 6e) was observed up to $250 \mathrm{MPa}$ and the Young's modulus at RT is estimated as $\mathrm{ELPSO}_{\mathrm{LP}}=58 \mathrm{GPa}$.

At $260 \mathrm{MPa}$, the $\{10 \overline{1} 1\}$ - and $\{10 \overline{1} 2\}$ - oriented planes (grains) parallel to the stress/extrusion direction lose their elastic linearity, which again indicates involvement of basal slip in strain accommodation. After yielding, the elastic strains corresponding to $\{10 \overline{1} 1\}-$ and $\{10 \overline{1} 2\}$ diffraction peaks are relaxed towards positive values from -5312 and $-5178 \mu$ strains at $255 \mathrm{MPa}$ to $-4381 \mu$ strains and $-3760 \mu$ strains at $292 \mathrm{MPa}$, respectively. Then, their values remain constant as a function of the applied stress, similar to the ED mode.

The behavior of the (0002) diffracted peak is completely different as compared to the ED mode. In contrast to microyielding phenomenon observed in ED, the deviation from the linearity of the $(0002)-\{10 \overline{1} 0\}$ pair, connected with the extension twinning, is not so pronounced. However, it is worthy to note that concurrently the intensity of the (0002) diffraction peak slightly increases. These facts confirm the activation of the extension twinning, although less significant than in the ED case.

\subsubsection{Compression along direction tilted by $45^{\circ}$ from ED to TD}

Figure $6 \mathrm{c}$ shows the evolution of elastic strains for sample compressed in the direction at $45^{\circ}$ between ED and TD. The compression stress-strain curve and the evolution of the integrated intensity of the $\{10 \overline{1} 0\}$ and (0002) peaks are also plotted. In this case, most of the non-DRX grains are oriented with their basal planes at $45^{\circ}$ to the compression direction. Thus, they are ideally oriented for the activation of the basal slip. Similar to the ED sample, the $\{4 \overline{22} 8\}$ 
diffraction peak of the LPSO phase (Fig. 6e) exhibits linear behavior up to $350 \mathrm{MPa}$. The Young's modulus at RT is the same as in both ED and TD samples: $E_{L P S O}=58 \mathrm{GPa}$.

The macroscopic yield stress was measured as $290 \mathrm{MPa}$. The Young's modulus at RT was estimated as $\mathrm{EMg}_{\mathrm{M}}=47 \mathrm{GPa}$ and the internal strains again behave linearly in the elastic regime. At $263 \mathrm{MPa}$, below the macroscopic yield stress, grains oriented with $\{10 \overline{1} 1\}-$ and $\{10 \overline{1} 2\}$ planes perpendicular to the compression axis lose their elastic linearity, which can be connected to activation of basal slip in these grains. Due to this fact, it is not possible to relate directly the beginning of the plastic deformation of the sample (macro yield stress) with the activation of the basal slip in these grains, as it is previously assumed in the compression along ED and TD. At $290 \mathrm{MPa}$, grains oriented with (0002) planes perpendicular to the compression axis lose their linearity, following a similar behavior to the sample compressed along ED. However, no relaxation phenomenon is observed in contrast to the ED case. The intensity of the (0002) diffraction peak increases slightly, but less than in the ED and TD modes (Figure 6d). Therefore, we assume that extension twinning control neither the beginning of the plasticity nor the plastic deformation during compression in the $45^{\circ}$ mode. This fact will be explained in detail in the discussion section.

\subsection{Clustering of the AE signal}

The raw AE signal recorded during compression of the ED sample is presented in Figure 7, as an example. The classical approach for evaluation of AE data includes extracting AE events by setting threshold level and hit definition time. However, this method fails, if distinguishing between the AE signals from various sources (dislocation slip, twinning and kinking) is required. Owing to their concurrent activity, the final signal is a sum of the contributions of these deformation mechanisms. Therefore, the adaptive sequential $k$-means (ASK) procedure for data evaluation was applied. This method, proposed recently by Pomponi and Vinogradov (Pomponi and Vinogradov, 2013), uses a non-supervised approach to AE signal categorization. The main steps of the analysis can be summarized as follows: 
1. A time window is defined, with which the continuously recorded data are sectioned into consecutive frames. The choice of the length always depends on the particular experiment and effects investigated. In this study, each frame contains 2048 samples at $1 \mathrm{MHz}$ sampling frequency, which means a $0.002 \mathrm{~s}$ long frame.

2. The power spectral density (PSD) function $G(f)$ is calculated for each frame by Welch's method(Welch, 1967). Furthermore, the normalized PSDs, defined as $\tilde{G}(f)=G(f) / E$, where $E$ is the AE energy per frame, is calculated as input parameters for statistical analysis of the signals. The normalized PSD have to fulfill the $\int_{0}^{\infty} \tilde{G}(f) d f=1$ condition.

3. The comparison between different normalized PSDs $\tilde{G}(f)$ is performed using the symmetric version of the Kullback-Leibler divergence. The first reference point for comparison is given by normalized PSD of the background noise. Every consecutive AE realization is then either assigned to the nearest cluster or used as a seed of a new cluster. The condition for new cluster forming follows the $k$-means algorithm.

4. Finally, the clusters are assigned to particular AE source mechanisms. In order to facilitate this step, several characteristic AE parameters are calculated, for example energy $E$ defined as $E=\int_{f_{\min }}^{f_{\max }} G(f) d f$ or median frequency $f_{m}\left(\int_{0}^{f_{m}} G(f) d f=\int_{f_{m}}^{\infty} G(f) d f\right)$.

The merit of this method is the determination of the source that governs the frequency spectrum, i.e. the dominant deformation mechanism in a given frame (time period). As a result, information about the dynamics of the system can be obtained.

When the clustering is completed, a dominant AE source mechanism is assigned to each cluster. There is no general rule for this procedure, since the characteristics of the clusters depend on many external parameters (examined material, sensor type, shape of specimens etc.). However, there are three basic steps that can be followed: 
1. Checking the time of the appearance of the events in a given cluster. For example, since the recording of $\mathrm{AE}$ data is always launched before the starting of the deformation test, the events in Cluster 1 naturally belong to the background noise.

2. Inspection of characteristic parameters of the PSDs, as energy, frequency distribution etc. For example, the background noise emits low energy signals in wide frequency range.

3. Comparison of the results with the supplementary experiments such a diffraction measurements or microscopy.

It is worthwhile to remark again that within a time window only one mechanism can be dominant. However, it does not mean that others AE sources are inactive. Only, they are simply less "loud".

Cross-plots of two characteristic parameters can facilitate the cluster assignment. Usually energy and median frequency are used for these purposes, since they well mirror the magnitude and speed of the source mechanisms. However, restricting the presentation of the cluster features to the two properties mathematically means a 2D projection of a multidimensional space. Thus, the overlapping of clusters is plausible.

The application of the ASK algorithm on the recorded AE data has resulted in the identification of 5 clusters. Energy - median frequency cross-plot, measured for all three orientations can be seen in Figure $8(\mathrm{a}-\mathrm{d})$. It is obvious that the plots are similar for all orientations. The time evolutions of the cumulative number of elements in the clusters are plotted in Figure 9(a-c) as a function of time. For comparison purpose, the compression test is also shown. Based on the results obtained during in-situ X-ray synchrotron diffraction experiments (section 3.2) and previous bibliography, the following source mechanisms were assigned to the clusters:

\subsubsection{Background noise (color code in figures - magenta)}

The identification of the source mechanism is obvious, since the elements in this cluster appear far before the launching of the straining. Since only a stressed material can emit AE (Kaiser, 1950), only the background noise can be responsible for AE. They are typically low energy and 
broad frequency range signals, as it is obvious in Fig. 8. As the straining starts, the number of elements in this cluster noise does not increase.

\subsubsection{Basal slip (color code in figures - blue)}

The number of events in this cluster starts to steeply increase immediately at the onset of deformation. Such a behavior can be connected with basal slip, which has a low critical resolved shear stress (CRSS) in magnesium alloys (Chapuis and Driver, 2011). Its activity has been observed far before the reaching the macroscopic yield (Vinogradov et al., 2013). The energy - median frequency plot of this cluster exhibits a characteristic drop shape in all the case (see as an example the ED case in Fig. 8b)(Horváth et al., 2016). At the initial stage of the straining the AE signals have higher energy, originated in densely populated dislocation avalanches. As the deformation progress, the mean free path for dislocation movement decreases owing to the increasing dislocation density. This fact induces a lowering of the energy and a shift of the median frequency to higher values.

The basal slip for all three sample orientations dominates rather at the beginning of the deformation, and its contribution to the frequency spectrum is soon suppressed by other mechanisms. Thus, the extent of basal slip during compression can be determined only from diffraction measurements.

\subsubsection{Twin nucleation (color code in figures - black)}

This cluster comes up almost concurrently with the basal slip. The majority of the elements in this cluster have higher energy and they lie in a narrower frequency range than previous signal. Nucleation of $\{10 \overline{1} 2\}\langle 10 \overline{1} 1\rangle$ extension twins can be assigned to this cluster. On one hand the CRSS of extension twinning is low (Chapuis and Driver, 2011) and thus this mechanism has been found active already at low applied stresses (Vinogradov et al., 2013; Máthis et al., 2015a). On the other hand, the twin nucleation is accompanied with quick reorientation of the crystal lattice, which results in emission of a large, burst-type elastic wave (Heiple and Carpenter, 1987). Consequently, the AE signals have high amplitude and energy. It is worth mentioning at 
this point that twin growth (i.e. increment in thickness) will not cause detectable AE (Heiple and Carpenter, 1987). The reason is given low energy wave emitted during slow motion of twin boundary (Gumbsch and Gao, 1999). For this reason, the combination of both AE and diffraction gives complementary information - about the nucleation (AE) and growth (diffraction). Muránsky et al. (Muránsky et al. 2010) found an unambiguous correlation between the sudden (0002) peak intensity change and the appearance of burst-like, high-energy AE signals for ZM20 magnesium alloy. Further, they showed that despite of the continuous increment of the (0002) peak intensity, the AE RMS voltage decreases after above the macroscopic yield. The "quietness" of the twin boundary moving was proved also by Lou et al. (Lou et al. 2007), who observed minimal AE activity in the unloading part of cyclic deformation tests, when the metallography showed a clear evidence of the detwinning.

\subsubsection{Non-basal slip (color code in figures - red)}

The ASK algorithm itself cannot distinguish between particular non-basal slip types. However, based on previous experimental data (Máthis et al., 2015a) and theoretical calculations (Mathis et al., 2015a) we can assume that particularly the prismatic and pyramidal $<a>$-slips contribute to this cluster. The energy and frequency distributions for this cluster are different for the particular orientations. In ED and TD, the non-basal signals are rather in low- and midfrequency range and the clusters have the expected tear-drop shape. In ND the amplitudes are significantly higher.

\subsubsection{Kinking (color code in figures - green)}

The signals in this cluster have similar character to that of twinning. However, its frequency is lower. This signal can be related to the kinking process in the LPSO phase, which is their characteristic deformation mode. Deformation kinks form, when a large stress acts parallel to basal slip planes. Avalanche-like motion of (0001) basal dislocations takes place accompanied by elastic buckling of planes (Hess and Barrett, 1949, Hagihara et al. 2016). During the ongoing loading, basal dislocations with opposite sign nucleate. They start to move against the 
dislocations from the first "wave", which leads to the kink formation. In summary, the kinking process is a highly correlated motion of large number of basal dislocations. Thus, the burst character and high energy feature is not surprising. Further, it is obvious that the extent of kinking significantly depends on the orientation of the samples, which is in good agreement with previous experimental observations (Garces et al., 2016).

3.4. Deformation experiments with in-situ X-ray synchrotron diffraction in a Mg alloy with a LPSO structure (MgY7Zn $\mathrm{Zn}_{5}$ alloy).

The kinking process cannot be observed easily in the in-situ diffraction experiments of the $\mathrm{MgY}_{2} \mathrm{Zn}_{1}$ alloy due to the low intensity diffraction peaks corresponding to the LPSO phase (see Fig. 3) and only the $\{4 \overline{22} 2\}$ diffraction peak can be studied. Therefore, similar in-situ diffraction experiments during compression tests were carried out in an alloy with a microstructure characterized by a high volume fraction of the LPSO phase (95\%) to understand in more detail the influence of the LPSO texture in the reinforcing capacity of the $\mathrm{MgY}_{2} \mathrm{Zn}_{1}$ alloy. The evolution of the internal strains in the axial direction corresponding to the $\{4 \overline{22} 2\}$, $\{4 \overline{22} 5\},(00018),\{4 \overline{22} 8\},\{30 \overline{3} 12\},\{4 \overline{22} 10\}$ and $\{60 \overline{6} 0\}$ diffraction peaks for the ED, TD and $45^{\circ}$ compression modes are shown in Figure 10(a-c). Moreover, similar to figure 6(a-c), the compression tests obtained during the in-situ diffraction experiments and the evolution of the integrated $\{4 \overline{22} 2\}$ and (00018) diffraction peaks in the axial direction as a function of the applied stress is also plotted.

The yield stresses calculated from Fig. 10(a-c) were 300, 201 and $198 \mathrm{MPa}$ for ED, TD and 45 modes, respectively. The values are smaller compared to the same alloy extruded at higher extrusion ratio (Garces et al., 2014). However, the relative mechanical behavior between the compression modes is the same. The extruded alloy is stronger when in compressed along the extrusion direction and it shows similar mechanical behavior in the TD and $45^{\circ}$ modes.

The evolution of the internal strains in the $\mathrm{MgY}_{7} \mathrm{Zn}_{5}$ alloy is more complex than the $\mathrm{MgY}_{2} \mathrm{Zn}_{1}$ alloy, since the plasticity is now controlled by the LPSO phase with a $18 \mathrm{R}$ rhombohedral crystal 
structure. Firstly, the evolution of the internal strains in the elastic regime differs for each diffraction peak indicating the elastic anisotropy of the LPSO phase, in contrast to the magnesium phase.

In all the cases, the lattice strains on $\{30 \overline{3} 12\}$-planes begins to deviate from the straight line on the elastic response. However, while this fact takes places in the vicinity of the macroscopic yield stress in the TD and $45^{\circ}$ modes $(200 \mathrm{MPa})$, the stress in the ED mode $(240 \mathrm{MPa})$ is lower than the macroscopic yield stress (around $300 \mathrm{MPa}$ ). It is important to mention than after compression, these grains remain under tensile residual stresses.

In the three cases, the evolution of the elastic strains for the $\{4 \overline{22} 2\}$ - and $\{60 \overline{6} 0\}$-planes oriented perpendicular to the compression axis exhibit a sigmoidal shape. Firstly, they behave elastically up to the applied stresses when $\{30 \overline{3} 12\}$-planes begin to yield. At this stress, the lattice strains on $\{4 \overline{22} 2\}$ - and $\{60 \overline{6} 0\}$-planes increase at a higher rate than in the elastic regime which indicates that these grains assumed an additional load transferred by the grains oriented with the $\{30 \overline{3} 12\}$-planes perpendicular to the compression axis . Finally, after the macroscopic yield stress, the evolution of the elastic strains changes again, and these grains yield at around 400, 300 and $300 \mathrm{MPa}$, for the ED, TD and $45^{\circ}$ compression, modes. The plastic deformation of both kinds of grains is accompanied by the slightly decrease in their integrated intensity. After unloading, these grains remain under compression residual stresses that emphasizes their reinforcing capacity.

\section{Discussion}

Previous studies demonstrated that the beginning of the plastic deformation in extruded Mg-Y-Zn alloys with a bimodal grain structure during a compression test along ED is controlled by the activation of the basal slip system only in the DRX grains (Garces et al., 2015a). In the non-DRX grains, which are oriented with their basal plane parallel to the extrusion direction, the Schmid factor for the basal slip (Fig. 11e) is near zero. Therefore, this slip system is 
completely inhibited and non-DRX grains deform by the activation of extension twinning system with a Schmid factor close to 0.5 (Fig 11h).

Our diffraction results also indicate that the beginning of plasticity is controlled by the activation of the basal slip not only in the ED but also in the other two compression cases. It is not surprising, since the random orientation of DRX grains increases the probability of the activation of this deformation mode. However, in the TD and 45 modes, the non-DRX can be also oriented favorably for slip in $<\mathrm{a}>$ basal system.

Figure 11a shows the stereographic projection of the $\mathrm{Mg}$ lattice for different orientations. In the ED mode, the compression axis is parallel to the $<1 \overline{1} 00>$ direction of the non-DRX grains (red point). For this orientation, the Schmid factor for the basal slip is zero, as it was commented previously. Thus, its activation is unlikely. In the TD mode, the compression axis is parallel to any direction contained $\{10 \overline{1} 0\}$ planes (Red line in Fig 11a.) of non-DRX grains. For grains with their $<0001>$ and $<11 \overline{2} 0>$ directions parallel to the compression axis, the Schmid factor for the basal system is zero (Fig. 11e.) and these grains cannot deform by the activation of this system. However, between these two perpendicular directions, there are possible orientations of non-DRX with non-zero Schmid factor values. Thus, coarse grains can deform by the activation of basal slip system. Since the importance of extension twinning in non-DRX grains is reduced, there is not a change of the initial texture(Fig. 6b). This fact is more evident in $45^{\circ}$ mode. The orientation of the non-DRX grains, in this case, is difficult to represent since there are multiple of possibilities. Figure 11(b-d) show three different possible orientations on the non-DRX grains. In all the cases, the Schmid factor is higher than 0.35 . Thus, it is expected the easy activation of the basal $<\mathrm{a}>$ system. Since the grain size is much larger in non-DRX grains (almost two orders of magnitude), the activation of basal $<a>$ system will occur rather in non-DRX than in the DRX grains. The Hall-Petch slope was calculated for this alloy by

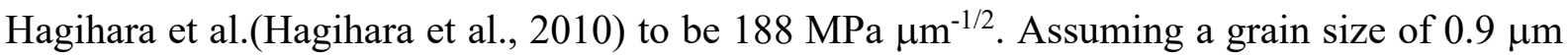
for the DRX grains and $10 \mu \mathrm{m}$ (the smaller dimension) for non-DRX grains, the Hall-Petch 
contribution would be 198 and $59 \mathrm{MPa}$, respectively. This fact can explain the loss of linearity of the elastic strains corresponding to $\{10 \overline{1} 1\}$ - and $\{10 \overline{1} 2\}$-diffraction peaks below the macroscopic yield stress (Fig. 6c). The non-DRX grains begin to deform before the DRX grains owing to their larger grain size. However, since their volume fraction is lower, the macroscopic yield stress is still controlled by the DRX grains.

Results of AE indicate that in the ED mode, the number of elements corresponding to the basal slip increases with the increasing stress up to $200 \mathrm{MPa}$, where the extension twinning takes over the dominancy in the frequency spectrum. In the TD mode, the dominancy of the basal slip lasts up to $260 \mathrm{MPa}$, which is in excellent agreement with the diffraction results. Moreover, the number of elements for basal slip is higher than that in the ED mode, which clearly signalizes the dominancy of the basal slip in the early stage of plasticity in TD direction. In the $45^{\circ}$ mode, the number of basal slip events increases from the onset of straining up to $100 \mathrm{MPa}$. Above this stress level there is a moderated twin nucleation followed by massive non-basal slip above the yield stress.

Extension twinning has been observed in all three compression modes, as it is demonstrated by increase of the intensity of (0002) peak (Fig. 6d). Obviously, it is more important in the ED mode, where the crystallographic $c$-axis of the non-DRX grains is perpendicular to the compression direction (Fig. 11a). Generally, the twinning is the most pronounced in $\mathrm{ED}$, followed by $\mathrm{TD}$ and $45^{\circ}$. The same conclusions can be drawn also from $\mathrm{AE}$ and EBSD measurements. First, the twinning cluster is more populated in ED direction than that in TD and $45^{\circ}$ (Fig. 9) and this dominancy in the plastic deformation is unambiguous (black lines in Fig. 9). It is worth to note that twin nucleation is present virtually during the entire test in $\mathrm{ED}$ and $\mathrm{TD}$, whereas in $45^{\circ}$ it terminates soon after reaching the yield point. Second, the orientation image maps (OIM) (Fig. 12-obtained by EBSD also confirms the presence of extension twinning for all sample orientations after whole test. In ED, the large non-DRX grains are fully reoriented after loading (Fig. 12a-see blue colored areas). The $c$-axes in those grains, 
which were initially perpendicular to the loading axis, are rotated toward ED during straining, as it is demonstrated by the illustration of hexagonal prisms on the OIM map (M. - mother orientation of the grain, tw. - orientation of the twins). This is a clear sign of $\{10 \overline{1} 2\}<10 \overline{1} 1>$ extension twinning, since during this mechanism the grains reorient by $86.3^{\circ}$. In $\mathrm{TD}$, however, the number of the grains which are favorably oriented for twinning is lower, i.e. only grains with the c-axis parallel to the compression axis. However, almost all grains, which undergo twinning, are fully reoriented. In $45^{\circ}$ mode, the twin growth is not so developed as in the previous two cases. Even after crack the majority of the favorably oriented grains are only partially twinned.

Combination of $\mathrm{AE}$ and diffraction gives detailed insight into other issue of the twinning process. Microplasticity caused by twinning was observed with diffraction measurement only for ED specimen (Fig. 6a - around $250 \mathrm{MPa}$ ). However, the AE results indicate that the nucleation of extension twinning in elastic regime takes place for all deformation direction. Nevertheless, only in ED is the twin growth large enough for influencing the diffraction pattern.

During the first steps of the extension twinning process, it has been observed a strong evolution of the elastic strains corresponding to the $\{10 \overline{1} 1\}$ - and $\{10 \overline{1} 2\}$ - diffraction peaks (Fig. 6) towards lower (in absolute values) strains. This effect is especially significant in the case of ED, where the volume fraction of twins is higher than in the other two cases. The relaxed strain is almost the double in ED than in TD and six times higher than in $45^{\circ}$ mode. It is interesting to note that a relaxation process has been previously reported in textures magnesium alloys during compression tests (Agnew et al. 2006; Clausen et al., 2008; Garces et al., 2012b; Muransky et al., 2010, Qiao et al., 2015). For example in extruded AZ31 alloy the elastic strains values corresponding to the $\{10 \overline{1} 1\}$ - and $\{10 \overline{1} 2\}$ - diffraction peaks change abruptly just after yield stress towards high negative values ( Clausen et al., 2008; Garces et al., 2012b). On the other hand, Muransky et al. (Muransky et al., 2010), in an extruded ZM20 alloy with fine grain size pointed out that elastic strains corresponding to the $\{10 \overline{1} 3\}$ - and $\{11 \overline{2} 0\}$ - diffraction peaks 
are inflected towards higher positive and negative values, respectively, similar to our case. This effect is either reduced, when dislocation slip controls compression deformation, or it is not present (during tensile test), which indicates its relationship with twinning process.

The evolution of the number of elements in the twinning cluster in ED direction increases monotonically up to $400 \mathrm{MPa}$ (approx. $60 \mathrm{~s}$ on time scale), where the second hardening stage starts (Fig. 8a). This is in good agreement also with the lowering of the intensity increment of (0002) (and decrement of $\{10 \overline{1} 0\}$ ) diffraction peak in Fig. 6a. In the $\{1010\} 45^{\circ}$ case, the texture change is moderate. However, on Fig 12c, the EBSD investigation revealed presence of extension twins. Accordingly, there is a sign of twinning activity in AE spectrum between 150 and $275 \mathrm{MPa}$ (cf. Fig. 9c), related with a microplastic regime. The intensity of the diffraction peaks corresponding to the twinning increases only from $275 \mathrm{MPa}$.

This apparent discrepancy between the AE and SD results can be elucidated as follows: owing to the initial texture the plasticity is rather controlled by non-basal slip (see discussion below). Thus, the contribution of this mechanism is dominant in the AE spectrum at the expense of that of other mechanisms (including twinning). Further, in contrast to ED, there is no twin nucleation in majority of DRX grains. Thus, their contribution to twinning cluster is missing. In TD, the evolution of the number of elements in the twinning cluster is similar to that in ED. It increases monotonically, but the magnitude is approximately 4 times smaller, since, as mentioned above, the number of the favorably oriented grains for twinning is also smaller. It is noteworthy that above $100 \mathrm{MPa}$ there is a small deviation from the ideal elasticity for the (0002) peak in Fig. 6b. This signalizes the growth of the nucleated twins. At the same stress level, the slope of the twinning curve in Fig. $9 \mathrm{~b}$ decreases, which indicates the termination of twin nucleation-propagation stage. This indicates the insensibility of the AE on twin growth.

The diffraction results from Fig. 6 provide only little information about the non-basal slips. As mentioned above, lattice strain on $\{10 \overline{1} 1\}$ planes can be partially connected with the prismatic $<a>$-slip. As it is obvious from Fig. 6, this mechanism is active for all three specimens. 
The AE indicates that the activity of the non-basal slips is the lowest in the ED sample. Only a weak increment above $450 \mathrm{MPa}$ can be observed. This can be explained by exhausting of twinning at this stress level, and its replacing by non-basal $<_{c}+a>$-slip (Máthis et al., 2015a). In $45^{\circ}$ and $\mathrm{TD}$ samples, the non-basal slip becomes the dominant deformation mechanism above yield point. This conclusion is in excellent agreement with the modeling results (Agnew et al., 2003), which predict crucial role of non-basal $<a>$-slip in the macroscopic yield. The highest AE activity of this mechanism, measured for the $45^{\circ}$ - specimen is evident, since in this case the twinning cannot considerably contribute to the strain accommodation.

It is important to point out that the orientation of the non-DRX grains cannot explain alone the sort of anisotropy observed in the present LPSO-containing alloy. This fact has also commented in recent studies in textured magnesium alloy in which a new dislocation density based approach is developed to incorporate the effects of propagation of dislocation trough twins generated during plastic deformation (Oppedal et al., 2013, Quio et al., 2017). In the

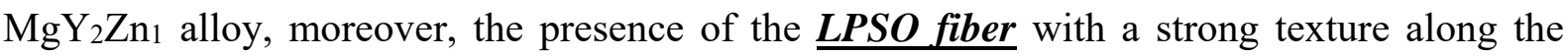
extrusion direction with a higher Young Modulus includes an additional parameter to take into account. On one hand, the LPSO phase exhibits a strong plastic anisotropy (Hagihara et al. 2010b, Garces et al., 2014). The compressive yield stress of the extruded LPSO phase is 554, 383 and $411 \mathrm{MPa}$ when they alloy is tested in the ED, TD and 45 loading modes, respectively. The same tendency in the yield stress was showed in figure 4. The reinforcing effect of the LPSO phase was found the most effective in the ED mode, where the highest yield strength value was observed. There are two contributions that must be taken in account to understand the reinforcing effect of the LPSO phase and its dependence with the compression axis.

On one hand, the LPSO fibers exhibit a strong texture of with the basal plane parallel to the extrusion direction (see Fig. 4c), similar to magnesium grains in the $\mathrm{MgY}_{2} \mathrm{Zn}_{1}$ alloy. However, magnesium grains with the basal extrusion texture are mechanically "weak" due to the activation of the tensile twinning when they are compressed along the extrusion direction. 
Contrary to this, in-situ diffraction experiments in the $\mathrm{MgY}_{7} \mathrm{Zn}_{5}$ alloy (Fig. 10) showed that LPSO grains do not deform by tensile twinning. The slight decrease in the intensity of the $\{4 \overline{22} 2\}$ diffraction peak is related with the deformation by kinking. The formation of kink band rotated slightly the LPSO grains with respect to their original orientation $\left(<30^{\circ}\right)$ (Yamasaki et al., 2013, Egusa et al., 2013) opposite to the rotation caused by tensile twinning $\left(86^{\circ}\right)$. When the alloy in compressed along the TD or $45^{\circ}$, the beginning of the plasticity occurs at lower stresses than in the ED and it was controlled by the easy activation of the basal slip in grains with the $\{30 \overline{3} 12\}$-planes perpendicular to the compression axis. Therefore, the reinforcing effect in lowerd than in the ED compression mode. At higher stresses, the evolution of the internal strains of the $\{4 \overline{22} 2\}$ diffraction peak also shows the deformation by kinking in agreement with Garces et al. (Garces et al., 2014)

On the other hand, if the alloy is considered as a short-fibre reinforced composite, the shear-lag model (Lilholt, 1991) can be applied for the estimation of load transfer from the matrix to the reinforcing phase. The stress increment caused by presence of reinforcement can be then expressed as:

$\Delta \sigma_{L T}=\sigma_{m}\left[\frac{(l+d) A}{4 l}\right] f+\sigma_{m}(1-f)$

where $\sigma_{m}$ is the yield stress of the matrix, $f$ is the volume fraction of the reinforcement, $l$ is the projection of the length of the fibers in the direction of the applied load, $d$ is the diameter of the fibers and aspect ratio $A=l / d$. According to this model, the fibres aligned with the compression direction add the highest contribution. As it is obvious, our experimental results are in good qualitative agreement with this prediction.

Furthermore

Effect of compression direction on plasticity of the LPSO phase can be clearly seen from the evolution of the axial internal strains for the $\{4 \overline{22} 8\}$ diffraction peak of the LPSO phase (Fig. 
6e). In TD direction, the LPSO phase deforms plastically already at $250 \mathrm{MPa}$. In the ED and $45^{\circ}$ mode elastic behavior was observed up to $350 \mathrm{MPa}$ (which was the detection limit owing to the large intensity of the neighboring (0002) $\mathrm{Mg}$ peak.

The AE results show that in the ED sample, the number of elements in the "kinking" cluster increases with increasing stress (Fig. 8a). After reaching $400 \mathrm{MPa}$, the curve steeply increases, and the kinking becomes the most significant source mechanism in the AE spectrum. At the same stress level, the twin associated texture change slow down, as it is evident from Fig. 5a. Figure 11 presenting the microstructure of the compressed sample shows a clearly kinked LPSO phase at high applied stresses. A similar feature was observed for an extruded $\mathrm{MgY}_{7} \mathrm{Zn}_{5}$ alloy, constituted principally by coarse grains of the LPSO phase and having the same crystallographic texture (Garces et al., 2014).

The TD and $45^{\circ}$ modes, the kinking in the LPSO phase is not really significant, most probably owing to the weak load transfer effect and due to the lower volume fraction of grains oriented with the $\{4 \overline{22} 2\}$ - and $\{60 \overline{6} 0\}$-planes perpendicular to the compression axis. However, the slight increment of number of kinking signals around $280 \mathrm{MPa}$ for TD (Fig. 9b) and at $300 \mathrm{MPa}$ for $45^{\circ}$ (Fig. 9c) can be observed, which is in good agreement with the lattice strain data.

There is a question, how reliable is the clustering method and the assignment of the clusters to the particular deformation mechanisms. In general, there is an agreement between the research groups using this method that the basal slip and the extension twinning are activated already at low stresses (Máthis et al. 2015, Vinogradov et al., 2015). Both cited works exhibit similar evolution of the basal slip and twinning clusters despite the completely different materials used. The frequency distribution of the signals is significantly influenced by the AE sensor and the sample geometry used. Thus, the direct comparison of the results is difficult. However, the general features of the particular clusters (drop shaped basal slip cluster, narrow frequency range - high energy twinning signals, broadband low energy noise etc.) repeat systematically (Máthis et al. 2015, Vinogradov et al., 2015, Horváth et al. 2016). The signal to-noise ratio in 
our case (cf. Fig. 6) is rather high. However, the applied analysis is generally capable to reveal the low amplitude signals even they are hidden in the background noise (Danyuk et al., 2013). Despite the basal slip origin of the kinking, the basal slip and the kinking have a completely different $\mathrm{AE}$ response. In the case of basal slip, the $\mathrm{AE}$ signal is rather continuous, caused by continuous movement of large number of dislocations and generation of new ones. In contrast, the kinking is characterized by highly correlated piecewise propagation of dislocation-pairs (Matsumoto et al., 2013), which is accompanied by burst signal type.

Deformation of the LPSO phase without kink formation was reported by Kim et al. (Kim et al. 2015). Their transmission electron microscopy investigations revealed a huge basal $<a>$ dislocation activity and formation of dislocation walls in the LPSO laths. In the ED sample, the number of element increases with increasing stress and after reaching $390 \mathrm{MPa}$ this tendency is boosted. This means that enough stress is accumulated for launching the kinking. Fig. 13 presenting the microstructure of the compressed sample shows a clearly kinked LPSO phase at high applied stresses. Similar feature was observed for an extruded $\mathrm{MgY}_{7} \mathrm{Zn}_{5}$ alloy, constituted principally by coarse grains of the LPSO phase and having the same crystallographic texture (Garces et al. 2014). A further evidence for the right interpretation of the AE data can be obtained with the comparison of the outcomes of the ASK analysis with the in-situ SEM measurements. In ED, the sample is stopped at 360,390 and $445 \mathrm{MPa}$ and in TD at 300 and $370 \mathrm{MPa}$, respectively. In Fig. 14 development of the microstructure of the LPSO phase and $\alpha-$ $\mathrm{Mg}$ during compression loading along ED and TD is presented. The $\alpha-\mathrm{Mg}$ is seen as a dark grey phase, while light grey or white phase corresponds to LPSO due to higher concentration of Y and $\mathrm{Zn}$ comparing to $\alpha-\mathrm{Mg}$. In case of deformation along $\mathrm{ED}$, at $360 \mathrm{MPa}$ the LPSO phase seems intact by deformation, which corresponds to the lattice strain evolution (Fig. 12a). At $390 \mathrm{MPa}$ (Fig. 14b) wedges and slip trace-like kink bands appear in the LPSO phase (marked by red arrows in highlighted areas). Formation of those low angle dislocation boundaries, i.e. plasticity of LPSO phase, develops with further compression up to $445 \mathrm{MPa}$ (Fig.12c). Similar 
evolution of the microstructure was reported by Hagihara et al. (Hagihara et al. 2013) and Yamasaki et al. (Yamasaki et al., 2013). They emphasise that the kink formation can take place through both basal- and prismatic $<a>$-slip. In the case of wedge formation, the basal $<a>$-slip dominates. Narrower, slip trace-like boundaries are present, when the lattice rotation leading to kinking is governed by prismatic $<a>$-slip (Yamasaki et al., 2013). It is noteworthy that the appearance of the kinking around $390 \mathrm{MPa}$ are in excellent agreement with the sudden increment of the kinking cluster determined by the ASK analysis (Fig. 9a). In the case of deformation along TD, the in-situ SEM revealed deformation in $\alpha-\mathrm{Mg}$ both at 300 to $370 \mathrm{MPa}$ (Fig.14d-e) The visualization of the plasticity would need application of TEM (cf. (Kim et al. 2015)), which is out of scope of this work. The dominancy of the plasticity of the $\alpha-\mathrm{Mg}$ corresponds to the ASK results, which shows prevalence of clusters corresponding to dislocation activity ("basal" and "non-basal") in Mg matrix (Fig. 9b).

\section{Summary}

The combination of in-situ synchrotron diffraction, scanning electron microscopy(SEM) and acoustic emission experiments during compression tests has been used to elucidate the effect of the orientation of the non-DRX grains and the LPSO phase with respect to the loading axis on both the plasticity and reinforcing capacity in the extruded $\mathrm{Mg}_{97} \mathrm{Y}_{2} \mathrm{Zn}_{1}$ alloy. The specimens were compressed along (i) extrusion direction; (ii) transverse direction and (iii) along a direction tilted by $45^{\circ}$ from $\mathrm{ED}$ to $\mathrm{TD}$.

The coarse, non-recrystallized grains underwent significant plastic deformation for all sample orientations. In the ED mode, since they are oriented with the basal plane parallel to the extrusion direction, they deform by $\{10 \overline{1} 2\}\langle 10 \overline{11}\rangle$ extension twinning that occurs before the macroscopic yield stress. In the other two compression modes, not only twinning but also dislocation slip takes place in non-DRX grains. AE results distinguished the activations of nonbasal slip systems after the macroscopic yield stress. 
Non-DRX textured grains cannot explain the sort of anisotropy observed in the present LPSOcontaining alloy alone and the LPSO fiber has also an influence on the plastic deformation. The extruded $\mathrm{Mg} 97 \mathrm{Y}_{2} \mathrm{Zn}_{1}$ alloy behaves as metal matrix composite where the LPSO phase acts as the reinforcement. Under compression in ED, the reinforcing effect of the LPSO phase (elongated along ED) is significant and the ED sample exhibits the highest mechanical strength. However, at high stress level, the LPSO phase is deformed owing to the activation of the kinking process that can be also detected by the AE measurement and in-situ SEM above 400 MPa.

\section{Acknowledgements}

BESSY (Berlin, Germany) and DESY (Hamburg, Germany) are kindly acknowledged for beamtime on the beamlines EDDI and P07, respectively. We kindly acknowledge the support of the EU during the measurements at BESSY and DESY. We should like to acknowledge financial support of the Spanish Ministry of Economy and Competitiveness under project number MAT2016-78850-R and the Czech Science Foundation under the contract 16-12075S.

\section{References}

Agnew, S.R., Yoo, M.H., Tome, C.N., 2001. Application of texture simulation to understanding mechanical behavior of $\mathrm{Mg}$ and solid solution alloys containing Li or Y. Acta Mater. 49, 42774289.

Agnew, S.R., Brown, D.W., Tome, C.N., 2006. Validating a polycrystal model for the elastoplastic response of magnesium alloy AZ31 using in situ neutron diffraction. Acta Mater. $54,4841-4852$.

Brandes, E.A., Brooks, G.B., 1997. Smithells Metals Reference Book. Sections 15-2 Butterworth-Heinemann, Oxford, UK. 
Chapuis, A., Driver, J.H., 2011. Temperature dependency of slip and twinning in plane strain compressed magnesium single crystals. Acta Mater. 59, 1986-1994.

Christian, J.W., Mahajan, S., 1995. Deformation twinning. Progress Mater. Sci. 39, 1-157.

Clausen, B., Tomé, C.N., Brown, D.W., Agnew. S.R., 2008. Reorientation and stress relaxation due to twinning: modeling and experimental characterization for Mg. Acta Mater. 56, 24562468

Danyuk, A., Merson, D., Vinogradov, A., New prospects to use acoustic emission during scratch testing for probing fundamental mechanisms of plastic deformation. 2013. In: Application of Contemporary Non-destructive Testing in Engineering, Portoroz, Slovenia, pp. $567-574$.

Egusa, D., Yamasaki, M., Kawamura, Y., Abe, E., 2013. Micro-Kinking of hte Long-Period Stacking/Order (LPSO) Phase in a hot-extruded $\mathrm{Mg}_{97} \mathrm{Zn}_{1} \mathrm{Y}_{2}$ alloy. Mater. Trans. 54, 698-702 Garces, G., Maeso, M., Todd, I., Pérez, P., Adeva. P., 2007. Deformation behaviour in rapidly solidified Mg97Y2Zn (at.\%) alloy. J. Alloy Compd. 432, L10-L14.

Garces, G., Oñorbe, E., Dobes, F., Pérez, P., Antoranz, J.M., Adeva, P., 2012a. Effect of microstructure on creep behaviour of cast $\mathrm{Mg}_{97} \mathrm{Y}_{2} \mathrm{Zn}_{1}$ (at.\%) alloy. Mater. Sci. Eng. A 539, 4855.

Garces, G., Oñorbe, E., Pérez, P., Klaus, M., Genzel, C., Adeva. P., 2012b. Influence of SiC particles on compressive deformation of magnesium matrix composites. Mater. Sci. Eng. A $533,119-123$.

Garces, G., Munoz-Morris, M.A., Morris, D.G., Jimenez, J.A., Perez, P., Adeva, P., 2014. The role of extrusion texture on strength and its anisotropy in a Mg-base alloy composed of the Long-Period-Structural-Order phase. Intermetallics 55, 167-176.

Garces, G., Morris, D.G., Muñoz-Morris, M.A., Perez, P., Tolnai, D., Mendis, C., Stark, A., Lim, H.K., Kim, S., Shell, N., Adeva P., 2015a. Plasticity analysis by synchrotron radiation in 
a $\mathrm{Mg}_{97} \mathrm{Y}_{2} \mathrm{Zn}_{1}$ alloy with bimodal grain structure and containing LPSO phase, Acta Mater. 94, 78-86.

Garcés, G., Requena, G., Tolnai, D., Pérez, P., Adeva, P., Jiménez, J.A., Stark, A., Schell, N., 2015b. Thermal expansion behaviour of Long-Period Stacking Ordered (LPSO) phase. Rev. Metal. 51 e043. doi: http://dx.doi.org/10.3989/revmetalm.043.

Garces, G., Perez, P., Cabeza, S., Lin, H.K., Kim, S., Gan, W., Adeva, P., 2015c. Reverse tension/compression asymmetry of a $\mathrm{Mg}-\mathrm{Y}-\mathrm{Zn}$ alloys containing LPSO phases. Mater. Sci. Eng. A 647, 287-293.

Genzel, C., Denks, I.A., Gibmeier, J., Klaus, M., Wagener, G., 2007. The materials science synchrotron beamline EDDI for energy-dispersive diffraction analysis. Nucl. Instr. Meth. Phys. Res. A 578, 23-33.

Gharghouri, M.A., Weatherly, G.C., Embury, J.D., Root, J., 1999. Study of the mechanical properties of Mg-7.7at.\% Al by in-situ neutron diffraction. Phil. Mag. 79, 1671-1695.

Gumbsch, P., Gao, H., 1999. Dislocations faster than the speed of sound. Science 283, 965968.

Hagihara, K., Kinoshita, A., Sugino, Y., Yamasaki, M., Kawamura, Y., Yasuda, H.Y., Umakoshi. Y., 2010a. Effect of long-period stacking ordered phase on mechanical properties of $\mathrm{Mg}_{97} \mathrm{Zn}_{1} \mathrm{Y}_{2}$ extruded alloy. Acta Mater. 58, 6282-6293.

Hagihara, K., Yokotani, N., Umakoshi, Y., 2010b. Plastic deformation behavior of Mg12YZn with 18R long-period stacking ordered structure. Intermetallics 18, 267-276

Hagihara, K., Fukusumi, Y., Yamasaki, M., Nakano, T., Kawamura, Y., 2013. Non-basal slip systems operative in $\mathrm{Mg}_{12} \mathrm{ZnY}$ long-period stacking ordered (LPSO) phase with $18 \mathrm{R}$ and $14 \mathrm{H}$ structures. Mater. Trans., 54, 693-697.

Hagihara, K., Mayama, T., Honnami, M., Yamasaki, M., Izuno, H., Okamoto, T., Ohashi T., Nakano, T., Kawamura, Y., 2016. Orientation dependence of the deformation kink band formation behavior in $\mathrm{Zn}$ single crystal. Int. J. Plast. 77, 174-191. 
Hammersley, A.P., 1997., FIT2D: An Introduction and Overview. ESRF Internal Report, ESRF97HA02T.

Heiple, C.R., Carpenter, S.H., 1987. Acoustic Emission Produced by Deformation of Metals and Alloys - A Review: Part I. J. Acoust. Em. 6, 177-204.

Hess, J.B., Barrett. C.S., 1949. Structure and Nature of Kink Bands in Zinc. T. Am. I. Min. Met. Eng. 185, 599-606.

Hirano, M., Yamasaki, K., Hagihara, K., Kawamura, Y., 2010. Effect of Extrusion Parameters on Mechanical Properties of $\mathrm{Mg}_{97} \mathrm{Zn}_{1} \mathrm{Y}_{2}$ Alloys at Room and Elevated Temperatures. Mater. Trans. 51, 1640-1647.

Horváth, K., Drozdenko, D., Máthis, K., Bohlen, J., Dobroň, P., 2016. Deformation behavior and acoustic emission response on uniaxial compression of extruded rectangular profile of $\mathrm{Mg}$ Zn-Zr alloy. J. Alloy Comp. 680, 623-632.

Inoue, A., Kawamura, Y., Matsushita, M., Hayashi, K., Koike, J., 2001. Novel hexagonal structure and ultrahigh strength of magnesium solid solution in the $\mathrm{Mg}-\mathrm{Zn}-\mathrm{Y}$ system. J. Mater. Res. 16, 1894-1900.

Itoi, T., Takahashi, K., Moriyama, H., Hirohashi. M., 2008. A high-strength Mg-Ni-Y alloy sheet with a long-period ordered phase prepared by hot-rolling. Scr. Mater. 59, 1155-1158.

Kaiser, J. 1950. Untersuchung über das Auftreten von Geräuschen beim Zugversuch. Fakultät für Maschinenwesen and Elektrotechnik. München: Technische Universität München.

Kawamura, Y., Kasahara, T., Izumi, S., Yamasaki. M., 2006. Elevated temperature Mg97Y2Cu1 alloy with long period ordered structure. Scr. Mater. 55, (2006) 453-456.

Kawamura, Y., Yamasaki. M., 2007. Formation and Mechanical Properties of $\mathrm{Mg}_{97} \mathrm{Zn}_{1} \mathrm{RE}_{2}$ Alloys with Long-Period Stacking Ordered Structure. Mater. Trans. 48, 2986-2992.

Kim, J.K., Sandlöbes, S., Raabe, D., 2015. On the room temperature deformation mechanisms of a Mg-Y-Zn alloy with long-period-stacking-ordered structures. Acta Mater. 85, 414-423. 
Kleiner, S., Uggowitzer, P.J., 2004. Mechanical anisotropy of extruded Mg-6\%Al-1\%Zn alloy. Mater. Sci. Eng. A 379, 258-263

Lilholt, H., 1991. Aspects of deformation of metal matrix composites. Mater. Sci. Eng. A 135, $161-171$

Lou, X. Y., Li, M,. Boger, R.K., Agnew, S.R., Wagoner, R.H., 2007. Hardening evolution of AZ31B Mg sheet. Int. J. Plasticity 23, 44-86.

Máthis, K., Csiszar, G., Čapek, J., Gubicza, J., Clausen, B., Lukáš, P., Vinogradov, A., Agnew, S.R., 2015a. Effect of the loading mode on the evolution of the deformation mechanisms in randomly textured magnesium polycrystals - Comparison of experimental and modeling results. Int. J Plasticity 72, 127-150.

Máthis, K., Čapek, J., Clausen, B., Krajňák, T., Nagarajan, D., 2015b. Investigation of the dependence of deformation mechanisms on solute content in polycrystalline $\mathrm{Mg}$ - $\mathrm{Al}$ magnesium alloys by neutron diffraction and acoustic emission. J. Alloy Compd. 642, 185-191.

Matsutomo, R., Uranagase, M., Miyazaki, N., 2013. Molecular dynamics analyses of deformation behavior of long-period-stacking-ordered structures. Mater. Trans., 54, 686-692. Muransky, O., Barnett, M.R., Carr, D.G., Vogel, S., Oliver. E.C., 2010. Investigation of deformation twinning in a fine-grained and coarse-grained ZM20 Mg alloy: Combined in situ neutron diffraction and acoustic emission. Acta Mater. 58, 1503-1517.

Oñorbe, E., Garcés, G., Pérez, P., Cabezas, S., Klaus, M., Genzel, C., Frutos, E., Adeva. P., 2011. The evolution of internal strain in $\mathrm{Mg}-\mathrm{Y}-\mathrm{Zn}$ alloys with a long period stacking ordered structure. Scr. Mater. 65, 719-722.

Oñorbe, E., Garcés, G., Pérez, P., Adeva, P., 2012. Effect of the LPSO volume fraction on the microstructure and mechanical properties of $\mathrm{Mg}-\mathrm{Y}_{2 \mathrm{X}}-\mathrm{Znx}$ alloys. J. Mater. Sci. 47, 1085-1093. Oñorbe, E., Garcés, G., Dobes, F., Pérez, P., Adeva, P., 2013. High-Temperature Mechanical Behavior of Extruded Mg-Y-Zn Alloy Containing LPSO Phases. Metal. Mater. Trans A 44, 2869-2883. 
Oppedal, A.L., El Kadiri, H., Tome, C.N., Vogel, S.C., Horstemeyer, M.F., 2013. Anisotropy in hexagonal close-packed structures: improvements to crystal plasticity approaches applied to magnesium alloy Philos. Mag. 93 4311-4330.

Pomponi, E., Vinogradov, A., 2013. A real-time approach to acoustic emission clustering. Mech. Syst. Signal. Pr. 40, 791-804.

Qiao, H. , Agnew, S.R., Wu, P.D., 2015. Modeling twinning and detwinning behavior of Mg alloy ZK60A during monotonic and cyclic loading. Int. J. Plast. 65, 61-84.

Qiao, H. , Guo, X.Q., Oppedal, A.L. El Kadiri, P.D., Wu, P.D., Agnew, S.R., 2017. Twin induced hardening in extruded Mg alloy AM30, Mat. Sci. Eng. A 687, 17-27.

Schindelin,J., Arganda-Carreras, I., Frise, E., Kaynig, V., Longair, M., Pietzsch, T., Preibisch, S., Rueden, C., Saalfeld, S., Schmid, B., Tinevez, J.Y., White, DJ., Hartenstein, V., Eliceiri, K., Tomancak, P., Cardona, A., 2012. Fiji: an open-source platform for biological-image analysis. Nature methods 9, 676-682.

Tane, M., Nagai, Y., Kimizuka, H., Hagihara, K., Kawamura, Y., 2013. Elastic properties of an Mg-Zn-Y alloy single crystal with a long-period stacking-ordered structure. Acta Mater. 61, $6338-6351$.

Vinogradov, A., Orlov, D., Danyuk, A., Estrin, Y., 2013. Effect of grain size on the mechanisms of plastic deformation in wrought $\mathrm{Mg}-\mathrm{Zn}-\mathrm{Zr}$ alloy revealed by acoustic emission measurements. Acta Mater. 61, 2044-2056.

Vinogradov A., Orlov, D., Danyuk, A., Estrin, Y., 2015. Deformation mechanisms underlying tension-copmpression asymmetry in magnesium alloy ZK60 revealed by acoustic emission monitoring. Mater. Sci. Eng. A. 621, 243-251.

Welch, P.D., 1967. Use of Fast Fourier Transform for Estimation of Power Spectra - a Method Based on Time Averaging over Short Modified Periodograms. Ieee Trans. Audio Electroacoustics. 15, 70-73. 
Yamasaki, M., Anan, T., Yoshimoto, S., Kawamura. Y., 2005. Mechanical properties of warmextruded $\mathrm{Mg}-\mathrm{Zn}-\mathrm{Gd}$ alloy with coherent $14 \mathrm{H}$ long periodic stacking ordered structure precipitate, Scr. Mater. 53, 799-803.

Yamasaki, M., Hashimoto, K., Hagihara, K., Kawamura. Y., 2011. Effect of multimodal microstructure evolution on mechanical properties of $\mathrm{Mg}-\mathrm{Zn}-\mathrm{Y}$ extruded alloy. Acta Mater. $59,3646-3658$.

Yamasaki, M., Hagihara, K., Inoue, S., Hadorn, J.P., Kawamura, Y., 2013. Crystallographic classification of kink bands in an extruded Mg-Zn-Y alloy using intragranular misoreintation axis analysis, Acta Mater., 61, 2065-2076.

Yoo, M.H., 1981. Slip, twinning, and fracture in hexagonal close-packed metals, Metal. Trans. A $12,409-418$.

\section{FIGURE CAPTIONS}

Figure 1(a-c). Scheme of orientation of both non-DRX grains (prolongation of the grains and orientation of hcp lattice) and fibers of LPSO phase with respect to sample geometry for the three compression modes: a) ED, b) TD and c) $45^{\circ}$. Fraction of the non-DRX grains with orientation of hcp lattice signed as "1" is larger than that with orientation " 2 " or " 3 " $\left(\mathrm{N}_{1}>\mathrm{N}_{2}\right.$, $\left.\mathrm{N}_{3}\right)$.

Figure 2(a-e). a) 3D microstructure of the extruded $\mathrm{Mg}_{97} \mathrm{Y}_{2} \mathrm{Zn}_{1}($ at.\%) alloy. b) Segmented and coloured $\mu \mathrm{CT}$-tomograms of $\mathrm{Mg}_{97} \mathrm{Y}_{2} \mathrm{Zn}_{1}($ at.\%) alloy. The magnesium matrix is transparent. c) Microstructure of the $\mathrm{Mg}$ alloy along the extrusion direction. Recalculated c) $10 \overline{1} 0$ and d) 0002 pole figures of the extruded $\mathrm{Mg}_{97} \mathrm{Y}_{2} \mathrm{Zn}_{1}($ at.\%) alloy. The extrusion direction is normal to the measured surface of the sample.

Figure 3(a-d). Synchrotron diffraction pattern as a function of the energy in the axial and radial directions in the $\operatorname{ED~(a,~b,~respectively)~and~TD~(c,d)~compression~modes~for~the~}$ $\mathrm{Mg}_{97} \mathrm{Y}_{2} \mathrm{Zn}_{1}($ at.\%) alloy before compression.

Figure 4(a-d). Debye-Scherrer synchrotron diffraction patterns obtained from the 2D detector for the $\mathrm{MgY}_{7} \mathrm{Zn}_{5}$ alloy in the a) $\mathrm{ED}$, b) $\mathrm{TD}$ and c) $45^{\circ}$ compression modes before compression 
tests. d) Synchrotron diffraction patterns in the axial direction as a function of the $2 \theta$ of the $\operatorname{MgY}{ }_{7} \mathrm{Zn}_{5}\left(\right.$ at.\%) alloy in the ED, TD and $45^{\circ}$ compression modes obtained from Fig. 4(a-c).

Figure 5(a,b). Compressive curves of the $\mathrm{Mg}_{97} \mathrm{Y}_{2} \mathrm{Zn}_{1}($ at.\%) alloy: a) continuous and b) in-situ discontinuous.

Figure 6(a-e). Compressive macroscopic stress-strain curve, axial internal elastic strains as a function of the applied stress and evolution of the integrated intensity of the major diffracted peaks for the magnesium phase during an in-situ compression test of the $\mathrm{Mg}_{97} \mathrm{Y}_{2} \mathrm{Zn}_{1}$ (at.\%) alloy for the a) ED, b) TD and c) $45^{\circ}$ compression modes. d) Integrated intensity as a function of the applied stress of the $\{10 \overline{1} 0\}$ and (0002) diffraction peaks in logarithmic scale for the three compression modes. e) Evolution of the axial internal strains as a function of the applied stress for the $\{4 \overline{2} \overline{2} 8\}$ diffraction peak of the LPSO phase for the three compression modes.

Figure 7. AE waveform stream measured for ED mode

Figure 8(a-d). Distribution of AE clusters in energy - median frequency space for a) ED, c) TD and d) $45^{\circ}$ compression modes. The clusters represented by different colors are assigned to particular source mechanisms. b) Cluster of the basal slip for ED compression mode

Figure 9(a-d). Time evolution of cumulative number of elements in the particular AE clusters assigned to background noise (magenta line), basal slip (blue line), twinning (black line); nonbasal slip (red line) and kinking (green line) for the a) ED, b) TD and c) $45^{\circ}$ compression modes. The dash lines represent the deformation curves as a function of time.

Figure 10(a-c). Compressive macroscopic stress-strain curve, axial internal elastic strains as a function of the applied stress and evolution of the integrated intensity of the major diffracted peaks for the rhombohedral 18R-LPSO phase during an in-situ compression test of the $\mathrm{Mg}_{88} \mathrm{Y}_{7} \mathrm{Zn}_{5}\left(\right.$ at.\%) alloy for the a) ED, b) TD and c) $45^{\circ}$ compression modes.

Figure 11(a-h). Stereographic projection of the magnesium lattice showing the crystallographic orientation of non-DRX with respect to the compression axis: (a-d). Schmid factors of nonDRX grains for the e) basal slip, f) non-basal slip, g) pyramidal slip and h) extension twinning for compression in the three modes (ED, TD and $45^{\circ}$ ).

Figure 12(a-c). Orientation Image Mapping (OIM) on the longitudinal section (the ED-TD plane) after compression in: a) ED, b) TD and c) $45^{\circ}$ modes.

Figure 13. Backscattered electron image showing the LPSO phase deformed by kinking after compression test of the alloy in the ED mode. 
Figure 14(a-e). In-situ SEM imaging during deformation along ED: a) 360MPa; b) $390 \mathrm{MPa}$; c) $445 \mathrm{MPa}$; and TD: d) $300 \mathrm{MPa}$; e) $370 \mathrm{MPa}$. 
a)

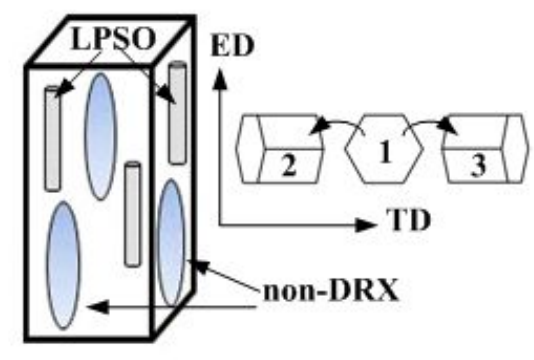

b)

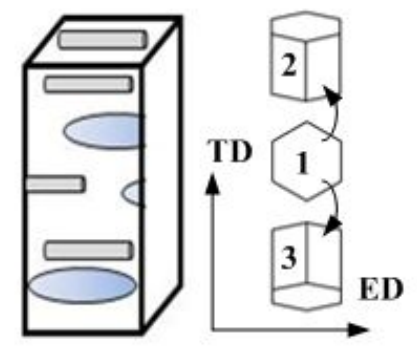

c)

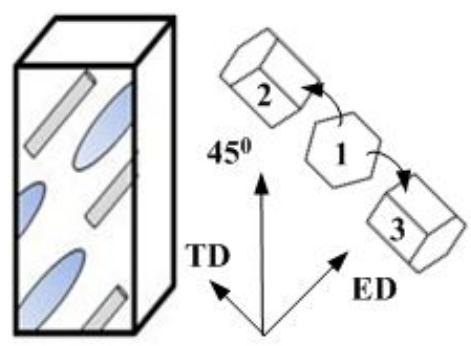

Figure 1 

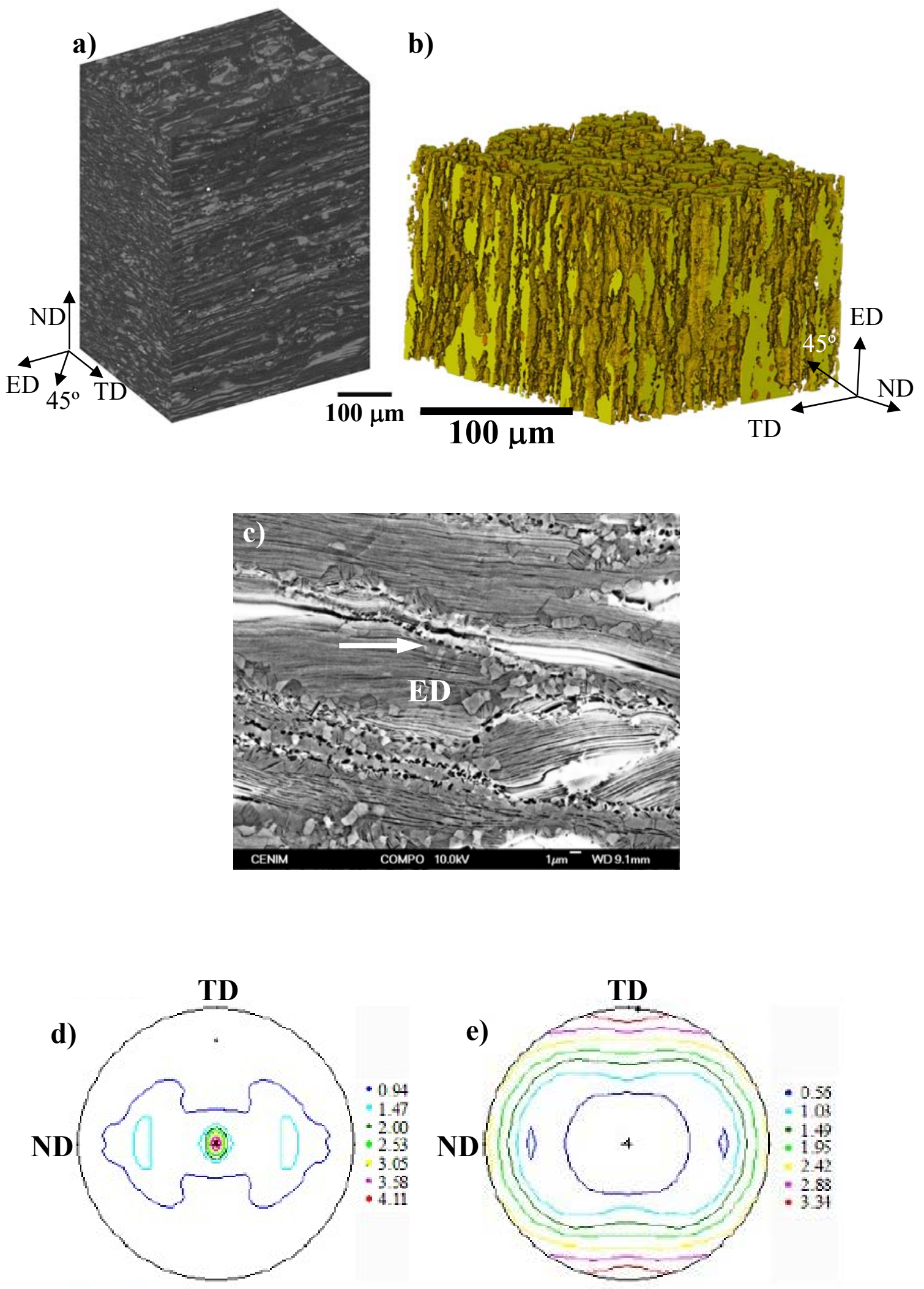

Figure 2.(a,e) 

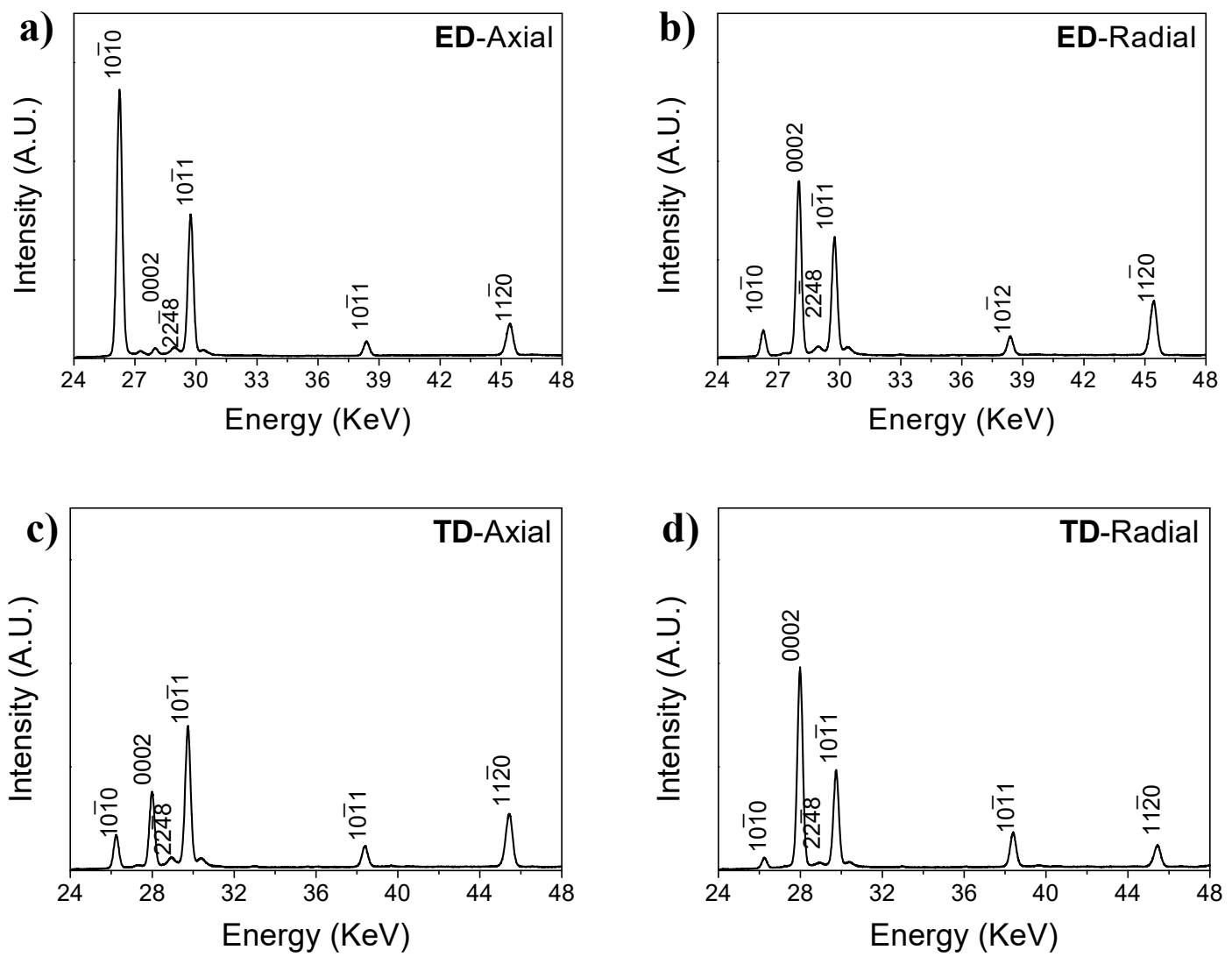

Figure 3 

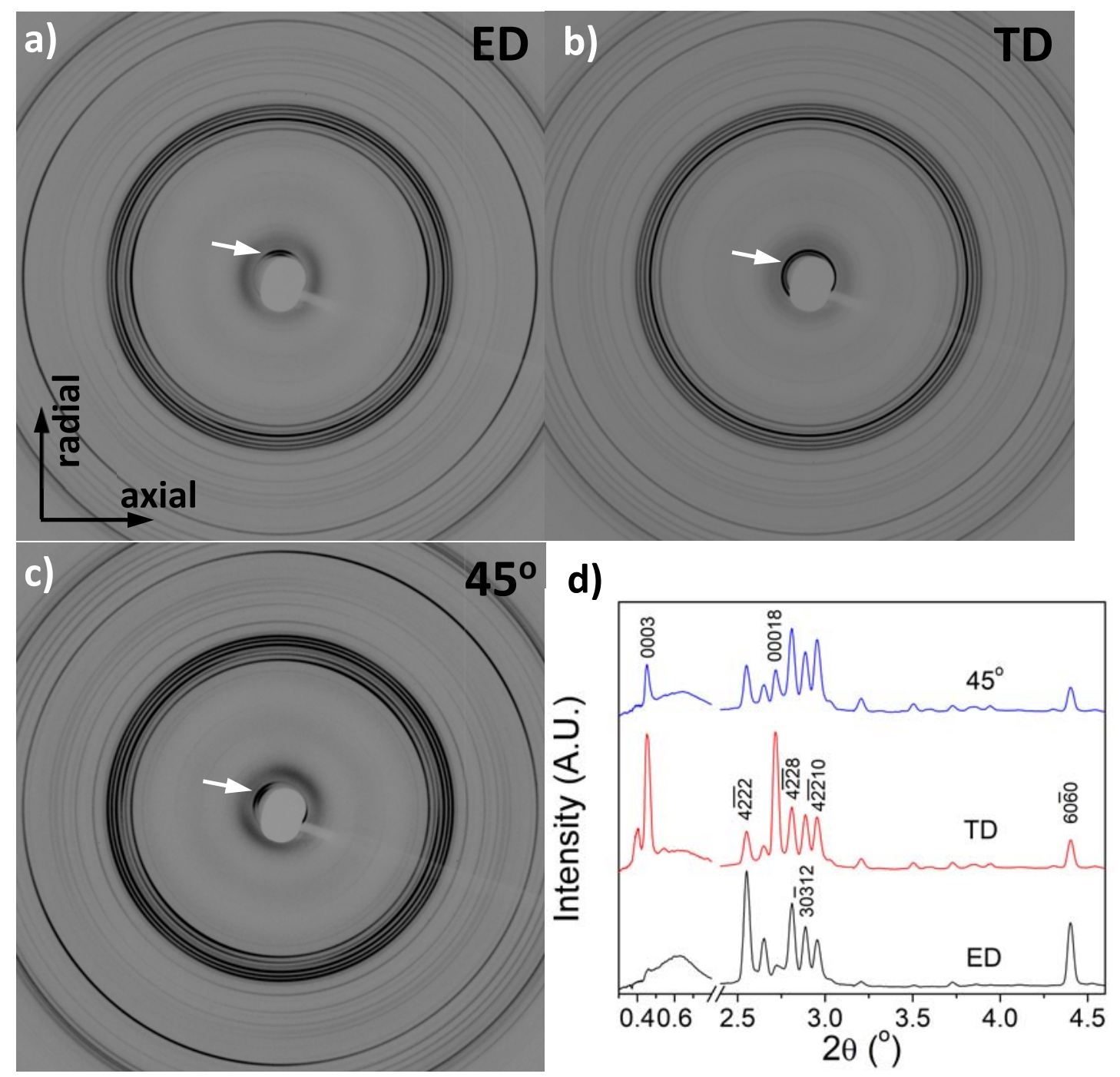

Figure 4 

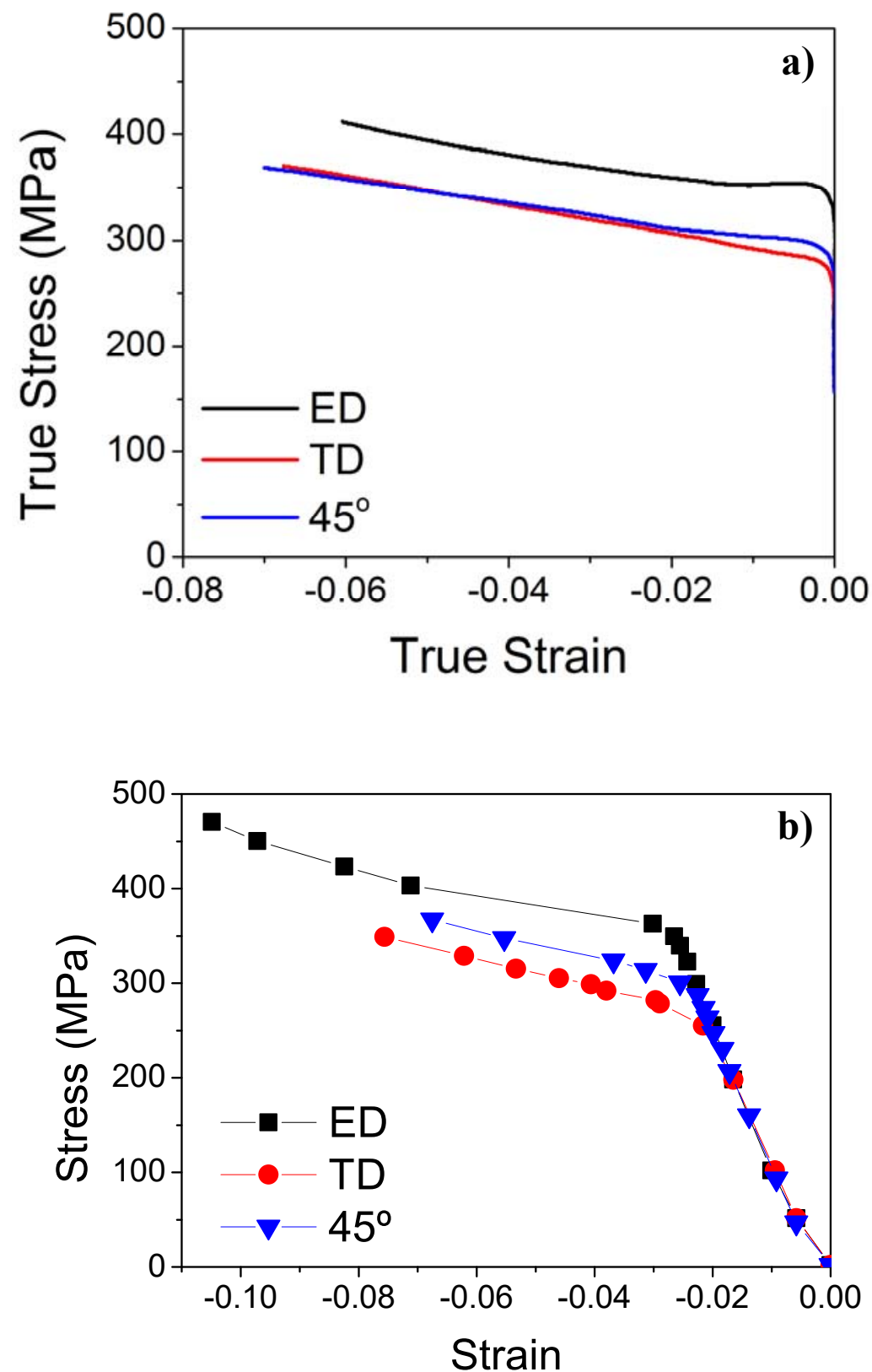

Figure 5(a,b) 

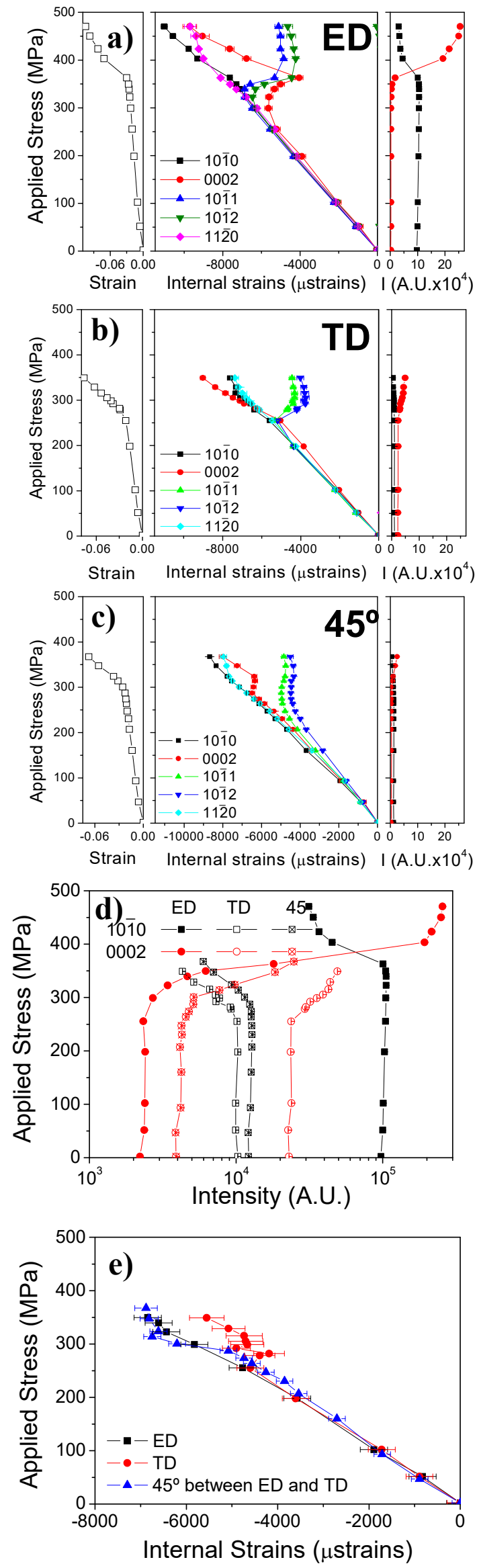

Figure 6 (a-e). 


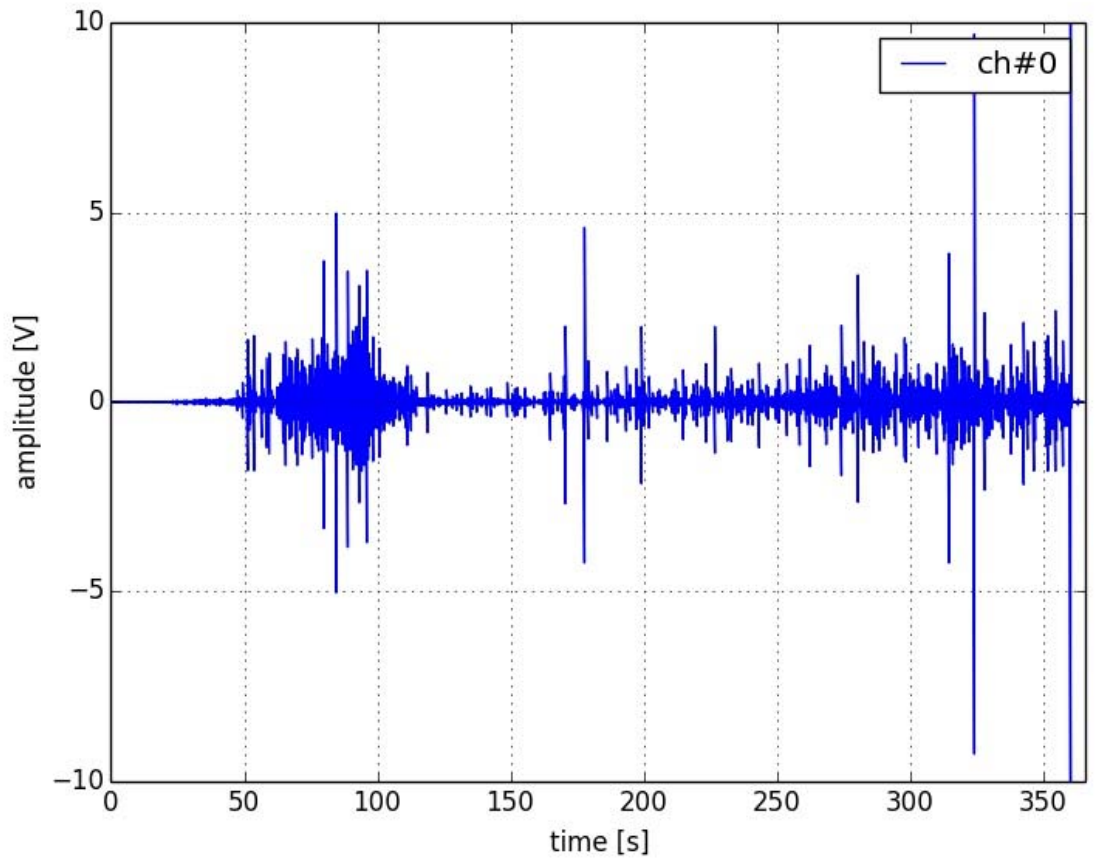

Figure 7 

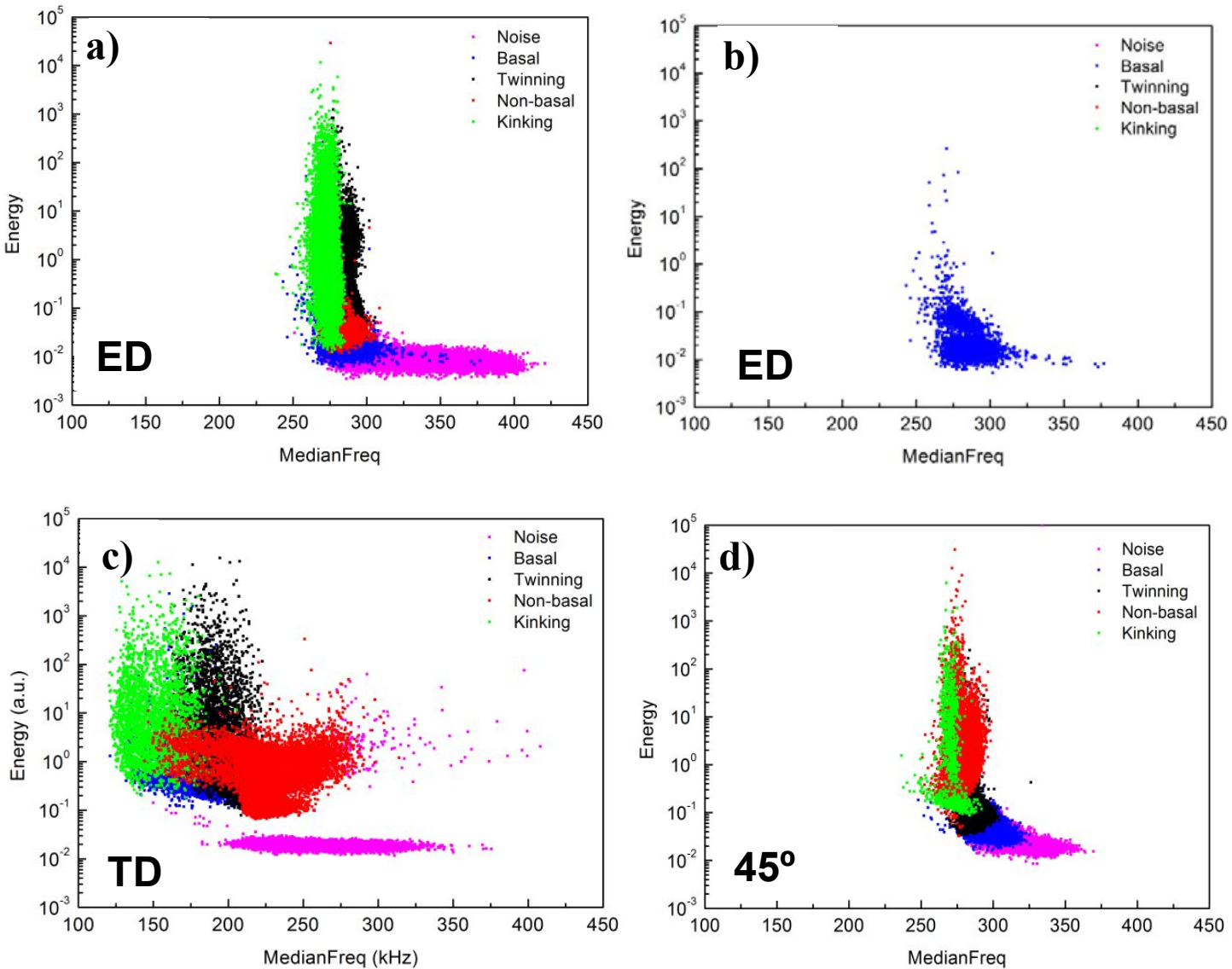

Figure 8 

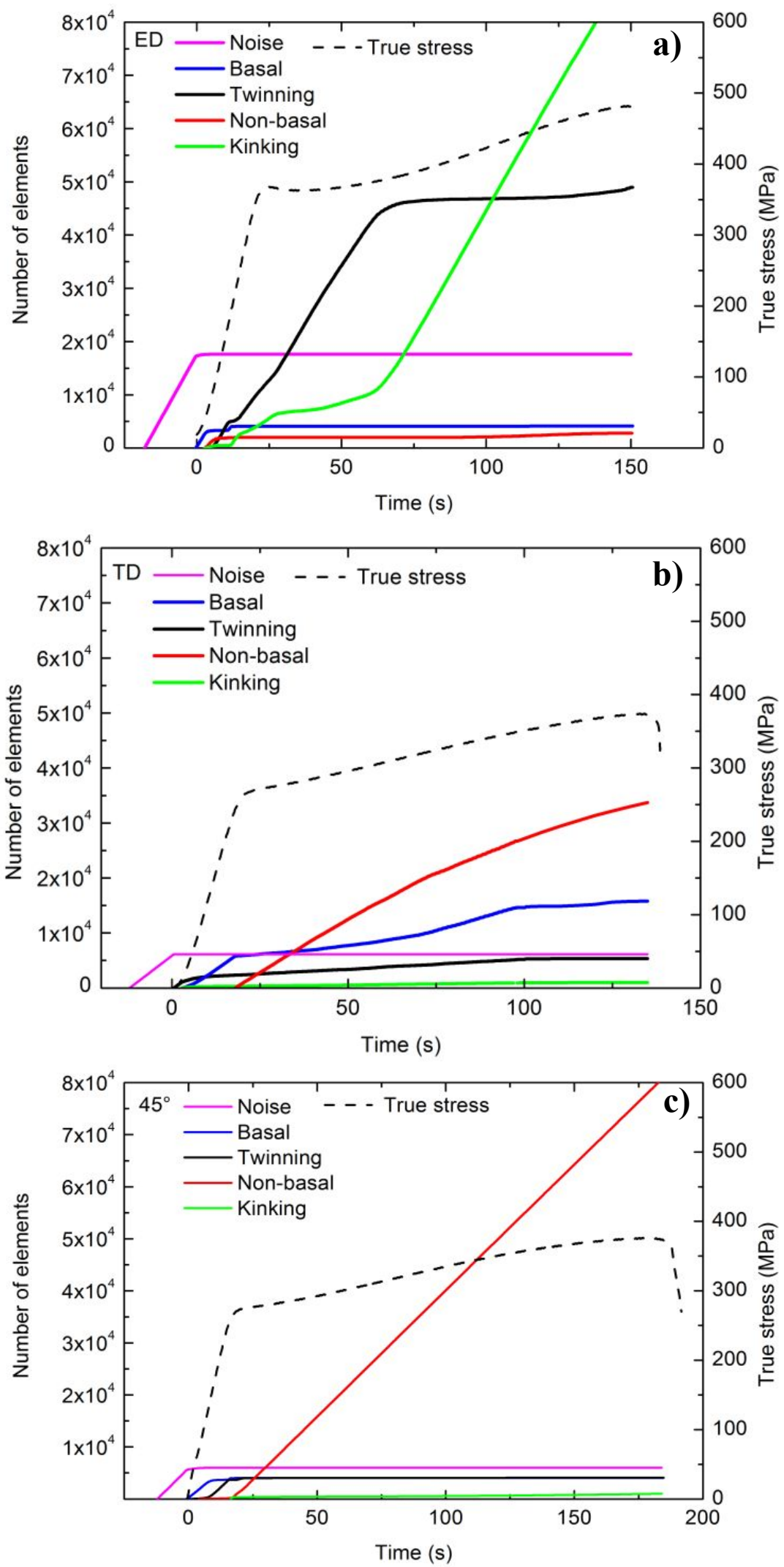

Figure 9(a-c) 

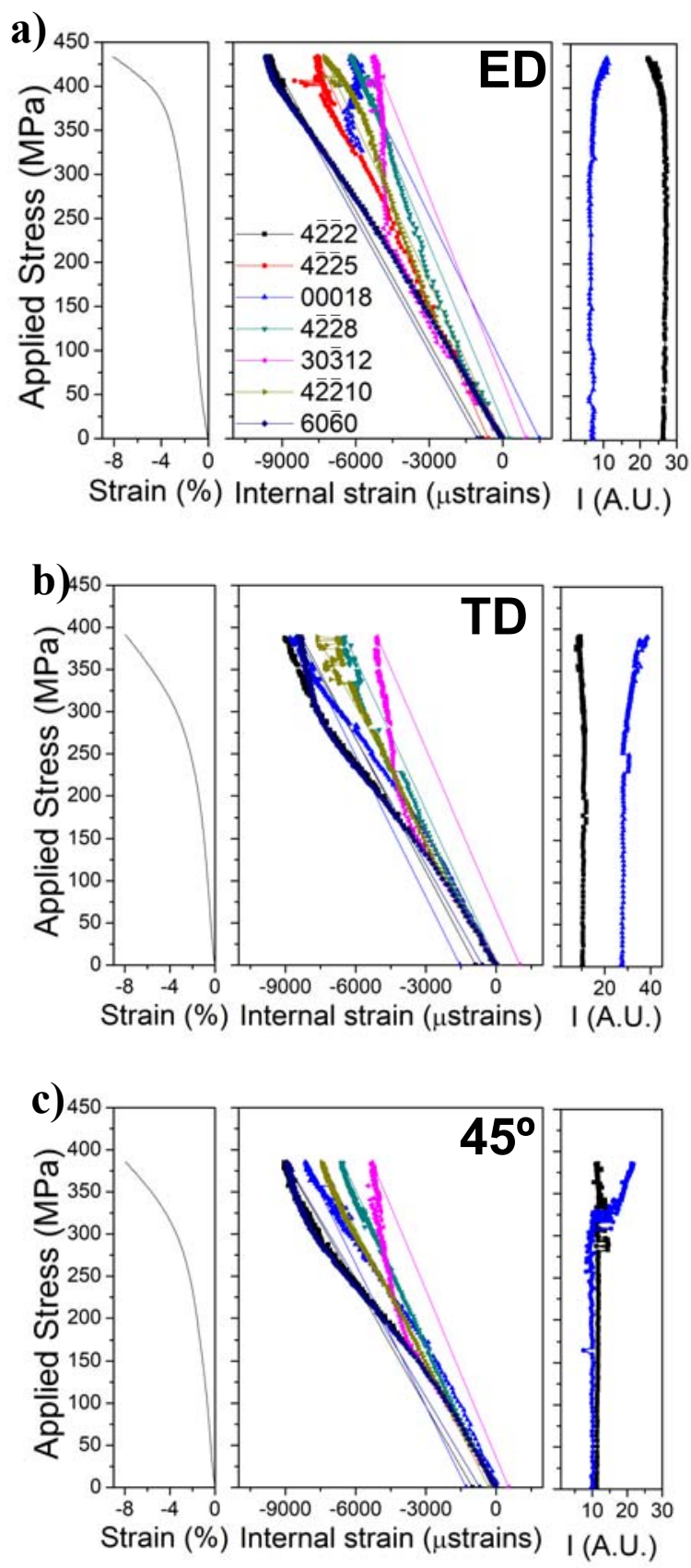

Figure 10(a-c) 

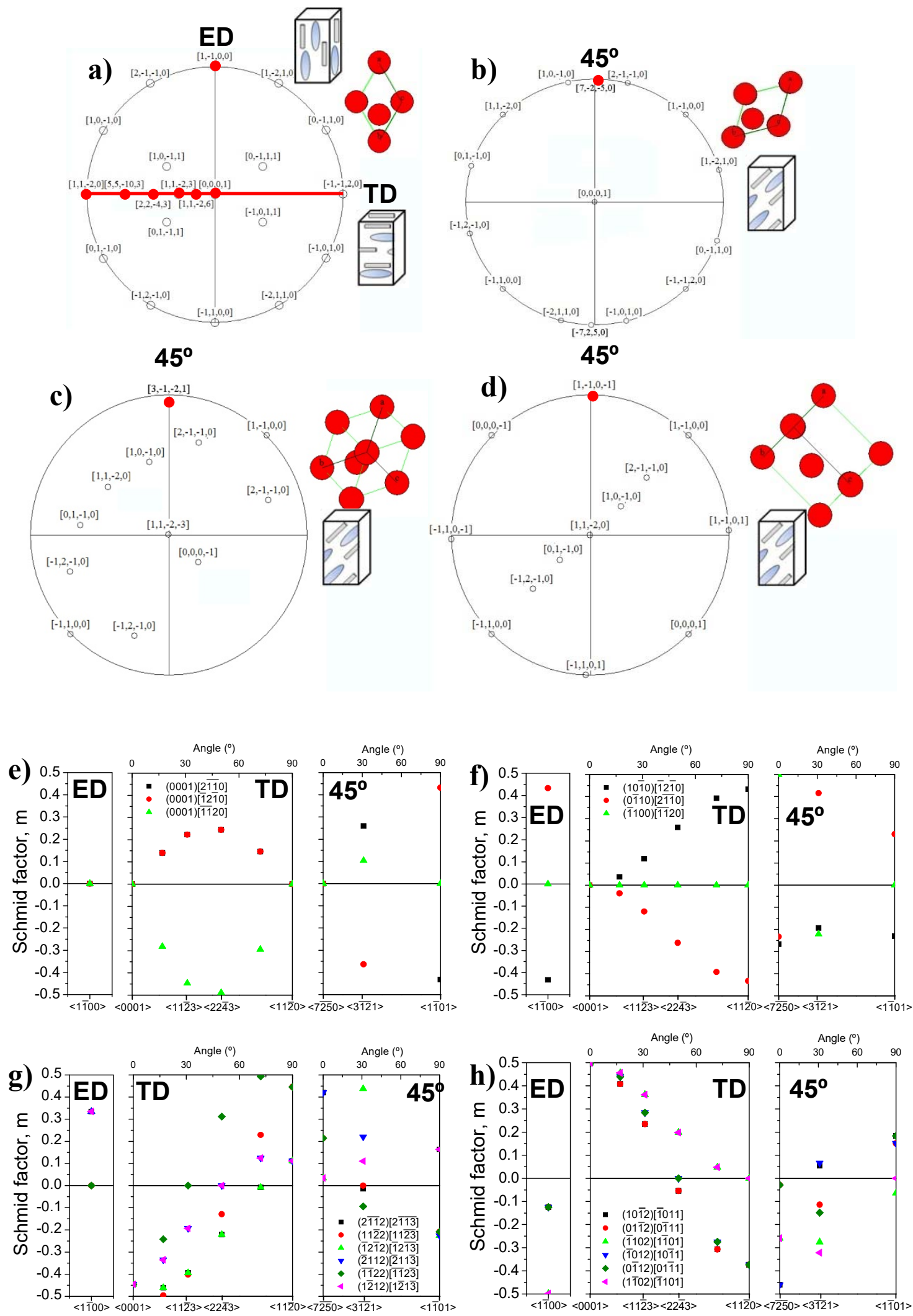

Figure 11(a-h) 


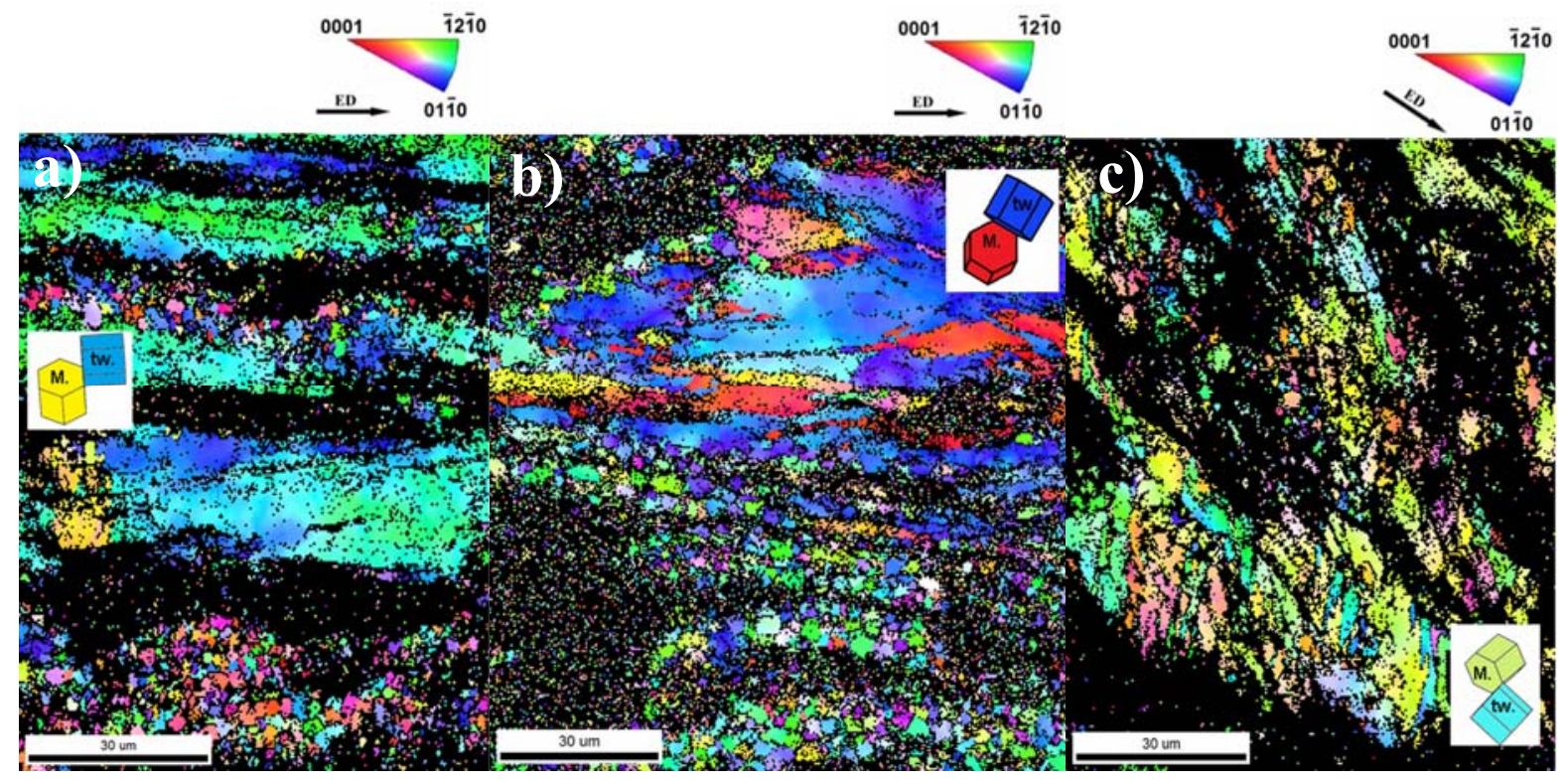

Figure 12(a-c) 


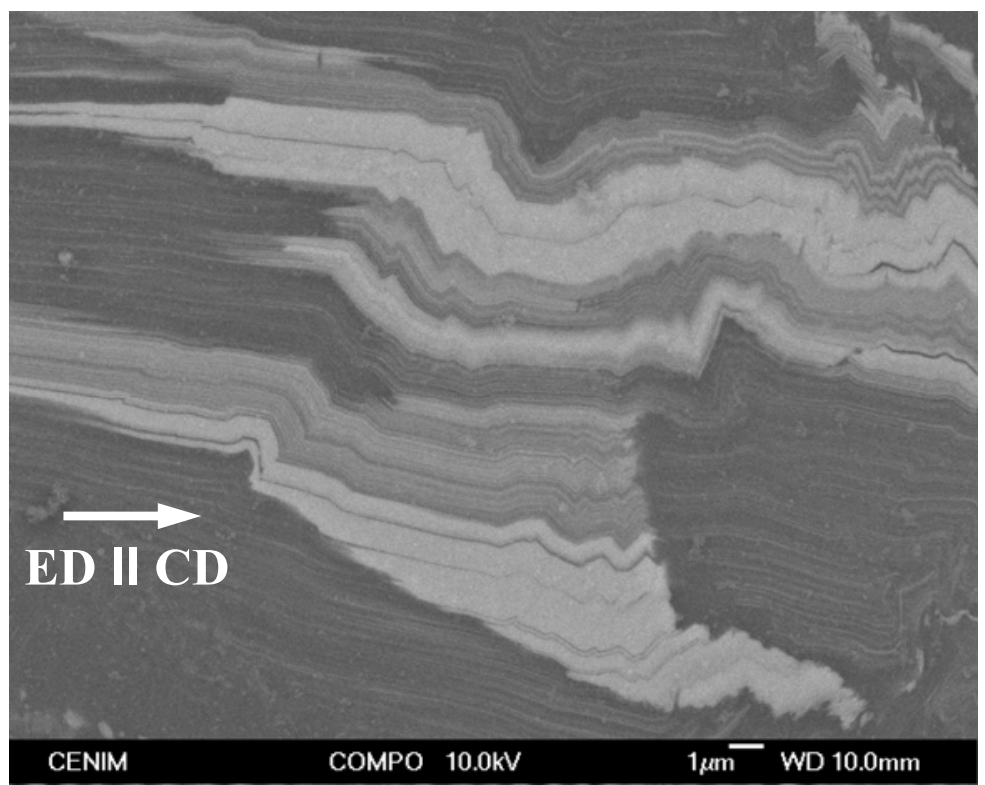

Figure 11 


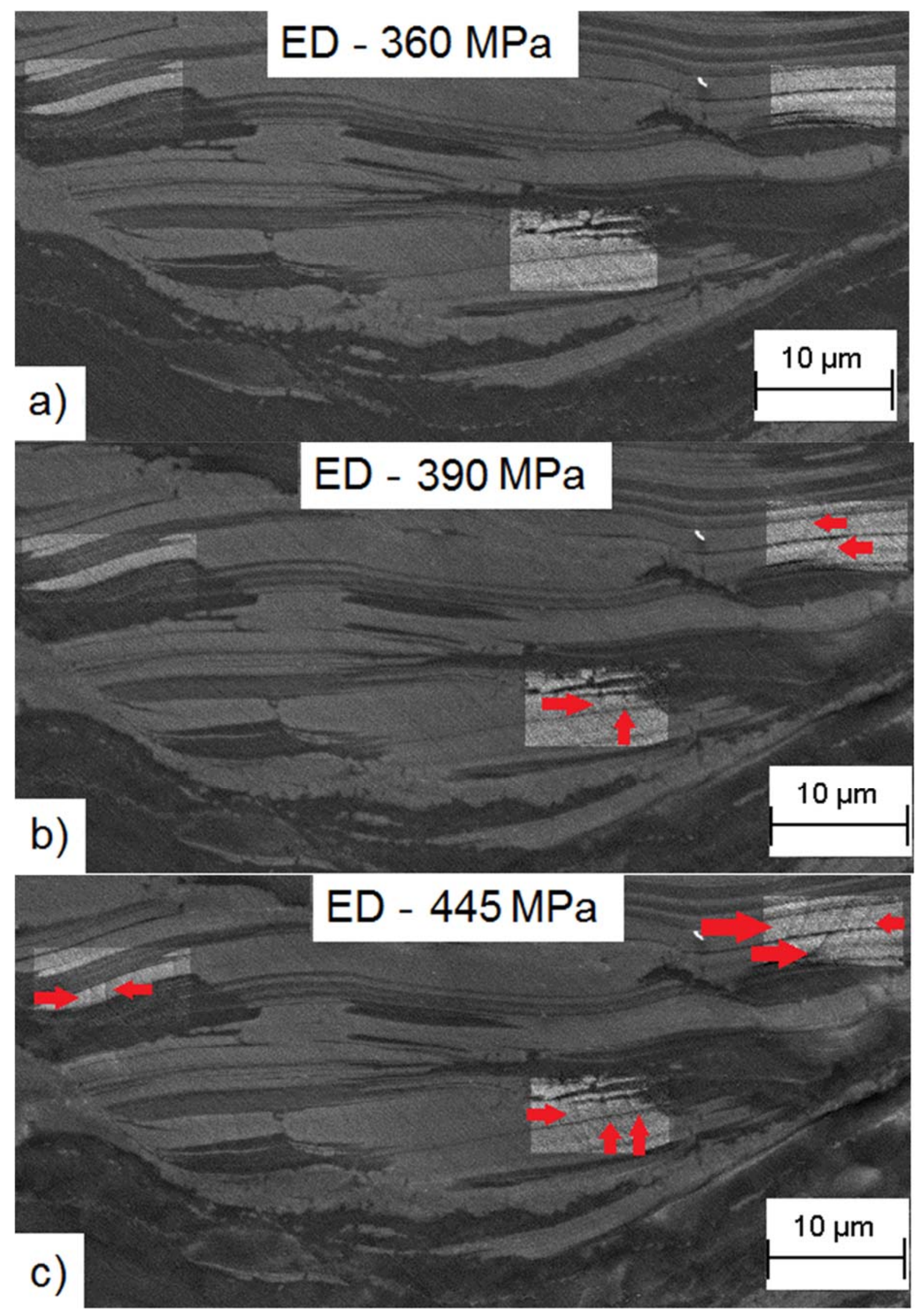




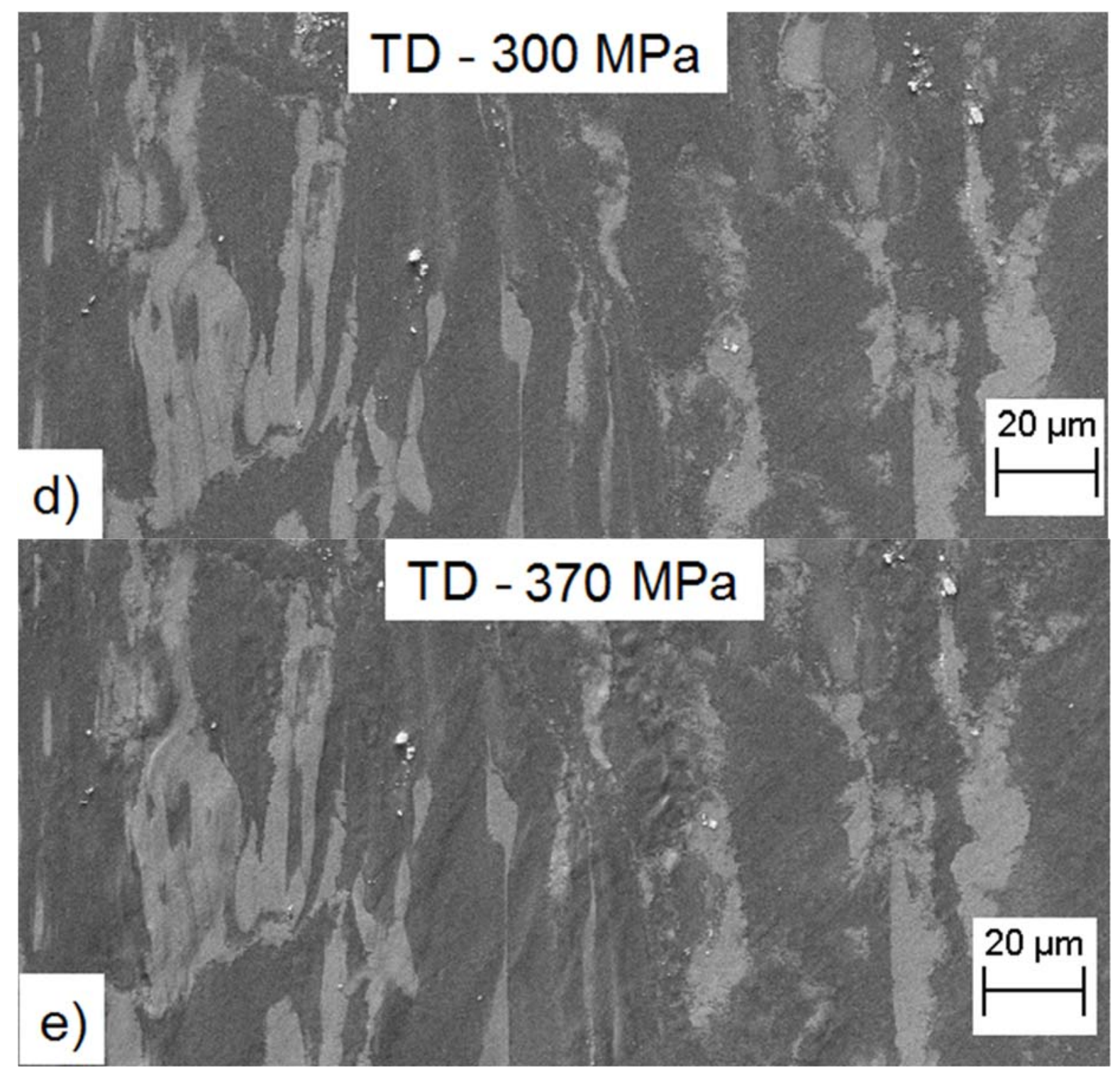

